\title{
Nonrigid Image Registration With Crystal Dislocation Energy
}

\author{
Yishan Luo and Albert C. S. Chung
}

\begin{abstract}
The goal of nonrigid image registration is to find a suitable transformation such that the transformed moving image becomes similar to the reference image. The image registration problem can also be treated as an optimization problem, which tries to minimize an objective energy function that measures the differences between two involved images. In this paper, we consider image matching as the process of aligning object boundaries in two different images. The registration energy function can be defined based on the total energy associated with the object boundaries. The optimal transformation is obtained by finding the equilibrium state when the total energy is minimized, which indicates the object boundaries find their correspondences and stop deforming. We make an analogy between the above processes with the dislocation system in physics. The object boundaries are viewed as dislocations (line defects) in crystal. Then the well-developed dislocation energy is used to derive the energy assigned to object boundaries in images. The newly derived registration energy function takes the global gradient information of the entire image into consideration, and produces an orientation-dependent and long-range interaction between two images to drive the registration process. This property of interaction endows the new registration framework with both fast convergence rate and high registration accuracy. Moreover, the new energy function can be adapted to realize symmetric diffeomorphic transformation so as to ensure one-to-one matching between subjects. In this paper, the superiority of the new method is theoretically proven, experimentally tested and compared with the state-of-the-art SyN method. Experimental results with 3-D magnetic resonance brain images demonstrate that the proposed method outperforms the compared methods in terms of both registration accuracy and computation time.
\end{abstract}

Index Terms-Convergence, dislocation, nonrigid registration.

\section{INTRODUCTION}

$\mathbf{N}$ ON-RIGID image registration is a crucial process for comparing or combining information available from different images. For medical image analysis in particular, it is a fundamental step for experts to observe the clinical evolution of a subject in a follow-up study or make comparison between different subjects. The non-rigid image registration problem can be described as follows. Given a reference image $I_{r}$ and

Manuscript received June 15, 2011; revised April 11, 2012; accepted May 25, 2012. Date of publication June 18, 2012; date of current version December 20, 2012. This work was supported in part by the Hong Kong RGC General Research Fund under Grant 612011 and the K. S. Lo Foundation, Hong Kong. The associate editor coordinating the review of this manuscript and approving it for publication was Prof. David S. Taubman.

The authors are with the Lo Kwee-Seong Medical Image Analysis Laboratory, Department of Computer Science and Engineering, Hong Kong University of Science and Technology, Kowloon, Hong Kong (e-mail: lisaluo@cse.ust.hk; achung@cse.ust.hk).

Color versions of one or more of the figures in this paper are available online at http://ieeexplore.ieee.org.

Digital Object Identifier 10.1109/TIP.2012.2205005 a moving image $I_{m}: \Omega$, where $\Omega \subset \mathbb{R}^{d}$ ( $d$ is the image dimension), the goal is to find a suitable transformation $\mathbf{h}$ such that the transformed moving image $I_{m}(\mathbf{x}+\mathbf{h})$ becomes similar to the reference image $I_{r}(\mathbf{x})$ [1]. Research on non-rigid image registration can be broadly divided into three areas.

1) Transformation model. Transformation model, which is the solution of the registration process, defines the spatial relationship between images. Both parametric transformation models (e.g. radial basis functions, B-splines) and non-parametric transformation models (e.g. linear elastic model, viscous fluid flow model and optical flow model) have been successfully applied in the field of image registration [2].

2) Similarity metric. The similarity metric measures the degree of alignment between images. It is usually an energy functional, which can take various forms according to different applications, for optimization.

3) Regularization term. The regularization term is used to obtain more likely solutions preferentially. Different kinds of prior knowledge are exploited to regularize the deformation field, such as the Tikhonov regularization term, the curvature term and the velocity field regularizer to achieve diffeomorphic mapping.

Generally, there is no universal combination of transformation model, similarity metric and regularizer applicable for all applications. The choice of each component is applicationspecific. Among the three components, similarity metric is probably the most critical element for a registration algorithm, as it defines the goal of optimization and measures how well the transformed moving image matches with the reference image. In general, there are two categories of similarity metric, i.e., feature-based metric and intensity-based metric.

1) Feature-based metrics rely on image features extracted from the images either automatically or interactively. The features can be points [3], [4], curves or surfaces [5], [6], and derived feature vectors [7]. Once extracted, the corresponding features are registered by minimizing a geometrical distance between them. The performance of feature-based metrics highly depends on the proper choice of features for the particular application and accurate localization of feature correspondences.

2) Intensity-based metrics are calculated directly from intensity values in the images rather than from geometric structures derived from the images. One of the simplest intensity-based metric is the sum of squared intensity differences (SSD), which is mainly designed for mono-modal image registration. SSD is based on the 
assumption that with optimal registration, two images only differ by the Gaussian noise. Because of its simplicity, SSD is widely used in many literatures [8]-[13]. However, exact intensity matching and Gaussian noise assumption are not always true in real cases, especially in inter-subject or inter-modal image registration. A less restrictive assumption is that corresponding intensities in the images have a linear relationship, cross-correlation (CC) is then applied [14], [15]. This metric helps register images whose intensity values can be related by a linear transformation. SSD and CC are mainly used to register images of the same modality. Because of the similarity of intensities in the images being registered, the subtraction and correlation techniques have an intuitive basis. However, in inter-modal image registration, the intensity statistical relationship between images is more complicated. Therefore, the information theoretic metrics are utilized, such as mutual information (MI) [16], [17], normalized mutual information (NMI) [18] and so on. This kind of metrics uses entropy as information measure and tries to maximize the amount of shared information in aligned images. These metrics state that the mutual information of the image intensity values of corresponding voxels is maximal if the images are geometrically aligned. As they make no explicit assumption on the nature of the relationship between intensity values in both modalities, they are widely used for multi-modal image registration.

The objective of this work is to provide a new choice of intensity-based similarity metric, which is inspired by the dislocation theory of crystal in physics [19]. When the atoms in a crystal are displaced from their perfect lattice sites, this defect is described as dislocation. The resulting distortion produces a velocity field in the crystal around the dislocation and the dislocation carries elastic energy. We make an analogy between object boundaries in images and the dislocations in crystals. When the boundaries are not in the optimal positions, an elastic field is produced, causing the boundaries to deform. Therefore, the registration process simulates a system in which the object boundaries of one image are deformed until they match the object boundaries in the other image. The optimal transformation is obtained by finding the equilibrium state, which corresponds to the minimum of the total potential energy associated with the boundaries in both images. The proposed method, namely registration with crystal dislocation energy, utilizes the elastic interaction between the two sets of boundaries in the reference image and the moving image to drive the registration process. In this new method, a voxelwise force deforming the moving image is calculated through the integral of the image gradient information over the entire image domain. In each iteration, the new method takes a more global and comprehensive view to find the correspondences between the reference image and the moving image, as compared with most of the current methods. As such, it helps find the correct correspondences and consequently the transformation relating the correspondences with relatively few number of iterations. Our proposed similarity metric mainly relies on the object boundaries in images to find the transformation between images, rendering it especially useful for the registration of the images with clear object boundaries or images with multiple structures, for example, human brain images. More distinctive boundary features will make the interaction between images much stronger, thereby efficiently drive the registration process. Moreover, the force driving the registration is an orientation-dependent and longrange interaction, making it applicable for large deformation. The proposed metric can be easily coupled with the symmetric diffeomorphic framework to realize diffeomorphic transformation. One predominant superiority of this new similarity metric is its fast convergence property. As the registration process is usually a computationally expensive procedure, fast convergence property thereby becomes especially important and useful. With fast convergence property, the proposed metric can achieve high registration accuracy within less number of iterations as compared with other metrics, thus reducing the computation time required for a registration process.

The rest of the paper is organized as follows. In Section II, the image registration framework is described based on the dislocation theory of crystal. The new method has been experimentally validated and compared with other related methods on real MR brain images, and results are presented in Section III. The paper concludes in Section IV.

\section{Methodology}

\section{A. Image Registration Methods Using Physics-Based Models}

Many concepts originate from physics-based models of materials which have been successfully applied in the field of image registration, such as, linear elasticity, fluid flow, optical flow and so on [2]. As the proposed method is also a physics-based model (i.e., the dislocation theory of crystal), the current physics-based models used in image registration are first briefly reviewed in this section.

1) Linear Elasticity Theory: The theory of linear elasticity is based on the notions of stress and strain. When a body is subject to an external force, the internal force within the body causes the body to deform. The body elasticity is represented by the following Navier-Cauchy PDE:

$$
\mu \nabla^{2} \mathbf{u}+(\mu+\lambda) \nabla(\nabla \cdot \mathbf{u})+\mathbf{f}=0
$$

where $\mathbf{u}$ is the displacement vector, and $\mathbf{f}$ denotes the external body force, which drives the registration process. Solving the Navier-Cauchy PDE is essentially an optimization problem that involves balancing external forces with internal stresses and smoothness constraints. This idea was introduced into image registration by Broit [20]. The transformation field can be obtained by solving the PDE.

2) Fluid Flow Model: The linear elasticity model has a major constraint that it only allows small deformations. However, large deformations typically reside in inter-subject registration. Then Christensen et al. [21] proposed a viscous fluid flow model for large deformation. The fluid flow model is based on the principle of continuum mechanics, which satisfies the conservation of mass, energy and linear momentum. Under these conditions, the Navier-Stokes equation for a 
compressible viscous fluid was derived as follows:

$$
\mu_{f} \nabla^{2} \mathbf{v}+\left(\mu_{f}+\lambda_{f}\right) \nabla(\nabla \cdot \mathbf{v})+\mathbf{f}=0 .
$$

Here $\mathbf{v}$ is related with the material derivative of the displacement $\mathbf{u}$. The Navier-Stokes equation describes the balance of forces acting in a given region of fluid. It characterizes an equilibrium state where changes in momentum of the fluid balance with the changes in pressure and dissipative viscous forces. The fluid flow model allows large deformations, but sometimes brings increasing registration error and intensive computation time [22].

3) Optical Flow: Optical flow model [23] has been widely used in computer vision and image processing to track small motions in time sequences of images. This is based on the principle of intensity conservation between image frames. The most well-known application of optical flow in image registration is the Demons algorithm proposed in [13]. It relates the displacement $\mathbf{u}$ to the change of intensity between the moving and fixed images and the spatial derivative of intensity in the fixed image, as follows:

$$
\mathbf{u}=\frac{\left(I_{m}-I_{r}\right) \nabla I_{r}}{\left(\nabla I_{r}\right)^{2}+\left(I_{m}-I_{r}\right)^{2}}
$$

\section{B. Numerical Framework of Nonrigid Image Registration}

In general, the objective of non-rigid image registration is to find the optimal transformation model $\mathbf{h}$ that maps each voxel in the moving image $I_{m}(\mathbf{x})$ to the reference image $I_{r}(\mathbf{x})$, so that the energy function which measures the difference between the moving image and the reference image can be minimized. The determination of the transformation model is a classic ill-posed problem and requires an additional regularization constraint. The solution to the non-rigid registration problem can be computed by optimizing the following energy function:

$$
E=\int_{\Omega} E_{\text {sim }}\left(I_{m}(\mathbf{x}+\mathbf{h}), I_{r}(\mathbf{x})\right) d \Omega+E_{\text {reg }} .
$$

The first term is the similarity metric and the second is the regularization term. In this paper, we focus on the first term and develop a new intensity-based similarity metric for monomodel image registration, which is proved to outperform the widely used SSD and CC metrics in terms of both registration accuracy and efficiency.

\section{Derivation of the Proposed Method}

The objective of non-rigid image registration in our concern is to match the corresponding objects of different images. The object boundaries, which are critical features to differentiate objects, take an important role during the registration process, as solving non-rigid image registration problem can be also considered as finding the corresponding features in two images. The object boundaries in the moving image are iteratively deformed towards the same object boundaries in the reference image until the two sets of boundaries are matched with each other in terms of both gradient magnitude and direction. In view of physics, if we treat object boundary as some elastic material, when the boundaries are not in the

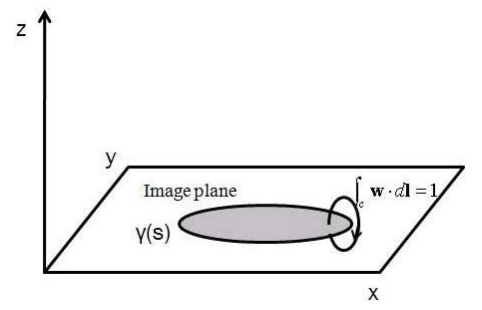

Fig. 1. 2-D image in the $z=0$ plane with the constraint that the integral $\int_{C} \mathbf{w} \cdot d \mathbf{l}=1$ along any curve $\mathrm{C}$ enclosing $\gamma(s)$.

optimal positions, the resulting distortion produces an elastic field. The boundaries in the elastic field carry some elastic potential energy. When placing the two sets of boundaries in a common space, according to the principle of minimum total potential energy, the boundaries move and interact so that the total energy associated with these boundaries is minimized and the equilibrium state is reached.

The proposed method is inspired by the dissipative dynamics of dislocations (line defects) in crystals of superconductivity, where the elastic energies are stored in the displacement field of the dislocations. These lines can move and interact to minimize the total energies until equilibrium. We use a 2D case to illustrate the idea. For a 2D image, the boundaries of the objects are curves (or line elements/segments). We define an energy associated with the curve similar to those in the dislocations. To facilitate the derivation of the new formulation, we place the plane containing the 2D images (the $x-y$ plane) into the $z=0$ plane of the 3D space, as shown in Figure 1. Considering a parameterized curve $\gamma(s)$ in 3D space, the elastic potential energy associated with $\gamma(s)$ is defined as,

$$
E(\gamma)=\min \int_{\Omega} \frac{1}{2}\|\mathbf{w}(x, y, z)\|^{2} d x d y d z,
$$

subject to the constraint enforced in the domain $\Omega$,

$$
\nabla \times \mathbf{w}(x, y, z)=\delta(\gamma) \boldsymbol{\tau}
$$

where $\mathbf{w}$, defined in the $3 \mathrm{D}$ space, is the stress acted on the curve and proportional to the elastic deformation of the curve. $\boldsymbol{\tau}$ is the unit tangent vector of $\gamma(s), \delta(\gamma)$ is the delta function of $\gamma(s)$ which is zero anywhere except on $\gamma(s), \nabla \times \mathbf{w}$ is the curl of $\mathbf{w}$. The meaning of Equation 6 is that the integral $\int_{C} \mathbf{w} \cdot d \mathbf{l}=1$ along any curve $C$ enclosing $\gamma(s)$. This constraint indicates the elastic field formed by the dislocations is a nonconservative field as the curve always stores some potential energy. The energy term in Equation 5 is defined based on the principle of minimum total potential energy, which is a fundamental concept used in physics, chemistry, biology and engineering. It asserts that a structure shall deform or displace to a position that minimizes the total elastic potential energy (i.e., $\int_{\Omega} \frac{1}{2}\|\mathbf{w}(x, y, z)\|^{2} d x d y d z$ in our case) to reach the equilibrium state. Equations 5 and 6 represent a simplified version of elasticity system associated with dislocations in solids [19], [24]. 
To solve the system, we apply the Lagrange method, the energy function then becomes,

$$
\begin{gathered}
E(\gamma)=\int_{\Omega}\left[\frac{1}{2}\|\mathbf{w}(x, y, z)\|^{2}+\lambda \cdot(\nabla \times \mathbf{w}(x, y, z)\right. \\
-\delta(\gamma) \tau)] d x d y d z,
\end{gathered}
$$

where the Lagrange multiplier $\lambda=\left(\lambda_{x}, \lambda_{y}, \lambda_{z}\right)$ is a vector function.

After taking variation with respect to $\mathbf{w}$ and $\lambda$, we have,

$$
\left\{\begin{array}{l}
\frac{\partial E}{\partial \mathbf{w}}=\mathbf{w}+\nabla \times \lambda=0 \\
\frac{\partial E}{\partial \lambda}=\nabla \times \mathbf{w}-\delta(\gamma) \tau=0
\end{array}\right.
$$

The first equation is equivalent to $\nabla \cdot \mathbf{w}=0$, and the second one is the constraint in Equation 6. This system for $\mathbf{w}$ can be solved analytically as [24],

$$
\mathbf{w}=-\frac{1}{4 \pi} \int_{\gamma} \frac{\mathbf{r} \times d \mathbf{l}}{r^{3}},
$$

where $\mathbf{r}=(x-x(s), y-y(s), z-z(s))$ is the vector between a point $(x, y, z)$ in the $3 \mathrm{D}$ space and a point $(x(s), y(s), z(s))$ on the curve $\gamma(s), r=\sqrt{(x-x(s))^{2}+(y-y(s))^{2}+(z-z(s))^{2}}$ is the distance between them and $d \mathbf{l}$ is a line element of the curve. Then the total energy associated with the curve is,

$$
E=\frac{1}{8 \pi} \int_{\gamma} \int_{\gamma^{\prime}} \frac{d \mathbf{l} \cdot d \mathbf{l}^{\prime}}{r}
$$

where $\gamma^{\prime}$ is the curve $\gamma$ using another parameter $s^{\prime}$.

Using the relationship $d \mathbf{l}=\boldsymbol{\tau} \delta(\gamma) d x d y d z$, we have

$$
E=\frac{1}{8 \pi} \int_{\Omega} \delta(\gamma) d x d y d z \int_{\Omega} \frac{\boldsymbol{\tau} \cdot \boldsymbol{\tau}^{\prime}}{r} \delta\left(\gamma^{\prime}\right) d x^{\prime} d y^{\prime} d z^{\prime},
$$

where $\mathbf{r}=\left(x-x^{\prime}, y-y^{\prime}, z-z^{\prime}\right)$ and $\boldsymbol{\tau}$ (or $\left.\boldsymbol{\tau}^{\prime}\right)$ is the unit tangent vector of $\gamma$ (or $\left.\gamma^{\prime}\right)$.

In line with the dislocation properties, if $\gamma(s)$ is a single loop or line, the energy is proportional to its length, as shown in Equation 10; if $\gamma(s)$ consists of multiple loops or lines, besides the energy proportional to the length, there is another contribution to energy because of the interaction of different loops or lines, i.e., the interaction between the elastic fields of different dislocations. As an example for the interaction energy, consider two curves $\gamma_{1}$ and $\gamma_{2}$, the total energy is,

$$
\begin{aligned}
E= & \frac{1}{8 \pi} \int_{\gamma_{1} \cup \gamma_{2}} \int_{\gamma_{1}^{\prime} \cup \gamma_{2}^{\prime}} \frac{d \mathbf{l} \cdot d \mathbf{l}^{\prime}}{r}, \\
= & \frac{1}{8 \pi} \int_{\gamma_{1}} \int_{\gamma_{1}^{\prime}} \frac{d \mathbf{l}_{\mathbf{1}} \cdot d \mathbf{l}_{\mathbf{1}}^{\prime}}{r}+\frac{1}{8 \pi} \int_{\gamma_{2}} \int_{\gamma_{2}^{\prime}} \frac{d \mathbf{l}_{\mathbf{2}} \cdot d \mathbf{l}_{\mathbf{2}}^{\prime}}{r} \\
& +\frac{1}{4 \pi} \int_{\gamma_{1}} \int_{\gamma_{2}} \frac{d \mathbf{l}_{\mathbf{1}} \cdot d \mathbf{l}_{\mathbf{2}}}{r},
\end{aligned}
$$

where $\gamma_{1}^{\prime}$ and $\gamma_{2}^{\prime}$ are $\gamma_{1}$ and $\gamma_{2}$ using other sets of parameters, $d \mathbf{l}_{1}$ and $d \mathbf{l}_{2}$ are respectively line elements of $\gamma_{1}$ and $\gamma_{2}$. The first two terms are self-energies of the two curves, which are proportional to their lengths. The third term is the interaction energy between them, which depends on their line directions, relative positions and lengths. The interaction energy between any two points on the two curves is proportional to $1 / r$, indicating the interaction will decay slowly as $r$ goes to infinity and may still interact between two long curves even if they are far from each other. In this sense, the interaction has a long capture range. Furthermore, the interaction is also orientationdependent as the curves are attractive when they have opposite directions and repulsive when they have the same direction. In summary, if two curves interact with each other, they move to minimize the total energy which is a combination of their self-energy and the interaction energy.

These formulations are developed based on the elasticity system associated with dislocations in crystals and this concept has been applied to solving image segmentation problem in [25]. As we have stated, non-rigid image registration can be considered as two sets of object boundaries moving and interacting to minimize the total associated energies, we use similar energy as above to achieve the goal of nonrigid image registration. We treat the object boundaries of the 2D image as the set of curves. Originally, within one image, each object interacts with other objects and deforms to find an equilibrium state. When the equilibrium state is reached, all objects stop deforming and produce objects with different shapes. When registering two images, the two images placed in a common space form a new elasticity system. The object boundaries of the two images interact with each other to reach a new equilibrium state. In such equilibrium state, the boundaries of the same object in the two involved images match each other in terms of boundary magnitude and direction.

Now we adapt the above dislocation energy formulation to solve the image registration problem. The object boundaries can be obtained from the gradient map of the graylevel images. The directions of the object boundaries of the reference image and the moving image are redefined as,

$$
\begin{aligned}
\boldsymbol{\tau}_{\boldsymbol{r}} & =\frac{\nabla I_{r}}{\left|\nabla I_{r}\right|} \times \mathbf{k}, \\
\boldsymbol{\tau}_{\boldsymbol{m}} & =-\frac{\nabla I_{m}}{\left|\nabla I_{m}\right|} \times \mathbf{k},
\end{aligned}
$$

where $\mathbf{k}=(0,0,1)$ is the unit vector in $z$ direction. The direction of the object boundary in the moving image is chosen to be opposite to that of the reference image. Thus the two images are attracted to match each other.

Using the dislocation interaction energy, together with the boundary directions defined for image registration as above, the new energy function for the registration problem is formulated as Equation 15. A detailed derivation for this formulation is provided in Appendix A.

$$
\begin{aligned}
E= & \frac{1}{8 \pi} \int_{\Omega} d \mathbf{x} \\
& \int_{\Omega} \frac{\nabla\left(I_{r}(\mathbf{x})-I_{m}(\mathbf{x}+\mathbf{h})\right) \cdot \nabla\left(I_{r}\left(\mathbf{x}^{\prime}\right)-I_{m}\left(\mathbf{x}^{\prime}+\mathbf{h}^{\prime}\right)\right)}{r} d \mathbf{x}^{\prime},
\end{aligned}
$$

where $\Omega$ is the whole image domain and $r=\left|\mathbf{x}-\mathbf{x}^{\prime}\right|$, which is the Cartesian distance between two points in $\Omega$. $\mathbf{x}$ and $\mathbf{x}^{\prime}$ are two coordinate representations in $\Omega$. This framework can also be extended for $3 \mathrm{D}$ images, in which the object 


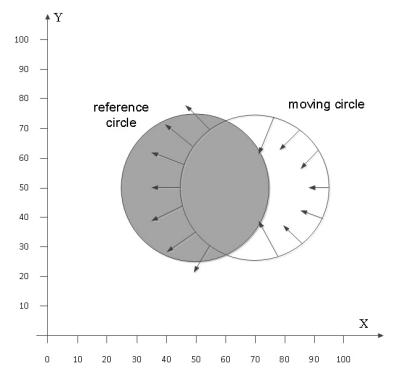

(a)

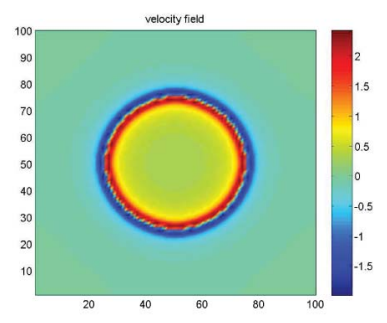

(b)

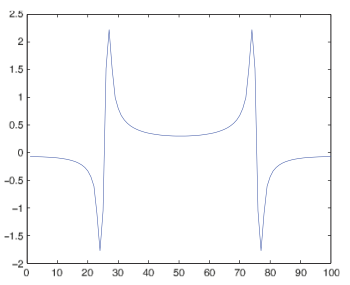

(c)

Fig. 2. (a) Reference disc object and the moving curve. The force acted on the moving curve is shown with arrows. (b) Normal velocity field generated by the reference image. (c) Normal velocity extracted from the line passing through the center of the disc $(y=50)$.

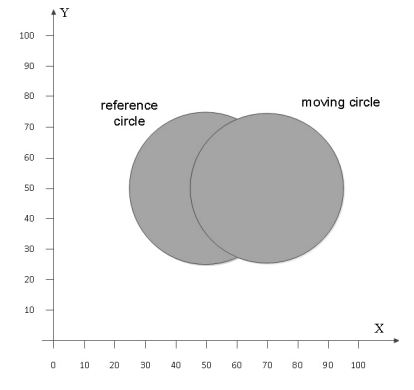

(a)

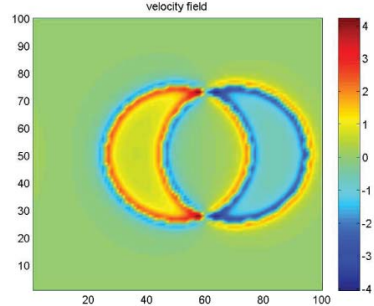

(b)

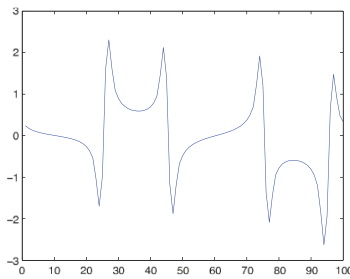

(c)

Fig. 3. (a) Two discs placed in the same space. (b) Normal velocity field. (c) Normal velocity along the line passing the center of the disc $(y=50)$.

boundaries become surfaces. For 3D images, the form of energy is,

$$
\begin{aligned}
E= & \int_{\Omega} \mathbf{d x} \\
& \int_{\Omega} \frac{\nabla\left(I_{r}(\mathbf{x})-I_{m}(\mathbf{x}+\mathbf{h})\right) \cdot \nabla\left(I_{r}\left(\mathbf{x}^{\prime}\right)-I_{m}\left(\mathbf{x}^{\prime}+\mathbf{h}^{\prime}\right)\right)}{r^{2}} d \mathbf{x}^{\prime} .
\end{aligned}
$$

For the concern of simplicity, all the following illustrations will still be presented with 2D example.

\section{Interaction Properties}

In this section, we use a synthetic example to show how the dislocation interaction drives the registration process. As shown in Figure 2(a), a disc object, which is darker inside (object with lower intensity values than background), is treated as the reference image and placed on the $x-y$ plane. We first show the normal velocity (force) field generated by the reference image alone by removing the contribution of the moving image in Equation A7, which is

$$
\mathbf{v}=-\frac{1}{4 \pi} \int_{\Omega} \frac{\mathbf{r} \cdot \nabla I_{r}}{r^{3}} d \mathbf{x}
$$

Figures 2(b) and (c) show the normal velocity field and the normal velocity along the line passing through the center of the disc. The magnitude of the velocity field is positive inside the disc and negative outside. If the moving curve is placed in the same plane as the reference disc, the force acted on the moving curve will attract it gradually towards the reference boundary, as illustrated in Figure 2(a). Positive $v$ means that the force is in the outward normal direction of the moving curve, i.e., $-\frac{\nabla I_{m}}{\nabla \nabla I_{m}}$. This indicates the interaction is orientation dependent. It can also be observed that the magnitude of velocity is strong near the object boundary and decays but never zero away from the boundary. This reflects the longrange property of the interaction, which still has effect on attracting the moving curve even when it is far from the reference curve. We also observe the interaction between two objects when they are placed in the same space with certain distance in between. Both the normal velocity field generated by the two objects and the normal velocity along the central line are shown in Figure 3. With Equation A7, we can see the interaction has two important features. The first is the interaction is stronger between more distinctive boundaries (larger gradient magnitude). This property is important in registration as it indicates that more distinctive features (boundaries in our case) contribute more than less distinctive features in driving the registration. We illustrate this property in Figure 4, where we increase the intensity difference between the object and the background so as to increase the gradient magnitude. Compared with Figure 3, the force magnitude is increased, as shown in Figures 4(b) and (c). Another important property of the interaction is that the force is proportional to $1 / r$ as stated in Section II-C. It means that the force is stronger when the two curves are closer. To show this property, we shorten the distance between two objects and obtain the velocity field, as shown in Figure 5. It is obvious that the velocity field is getting stronger as compared with Figure 3, which will make the two objects attract to each other in a much faster pace. 


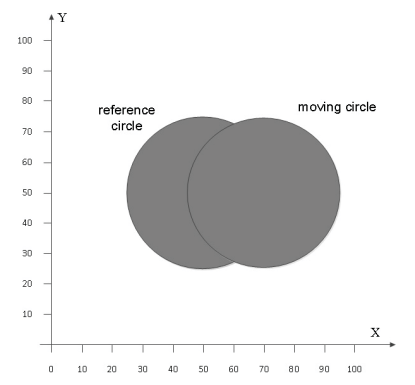

(a)

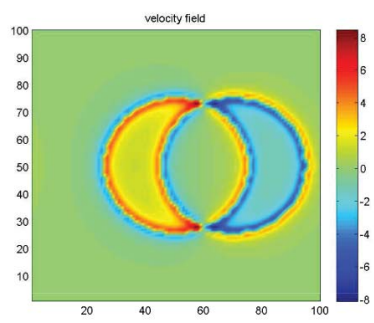

(b)

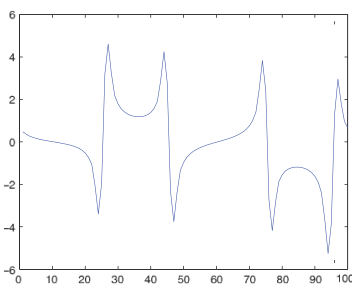

(c)

Fig. 4. (a) Two discs with larger gradient magnitude than those in Fig. 3. (b) Normal velocity field. (c) Normal velocity along the line passing the center of the disc $(y=50)$.

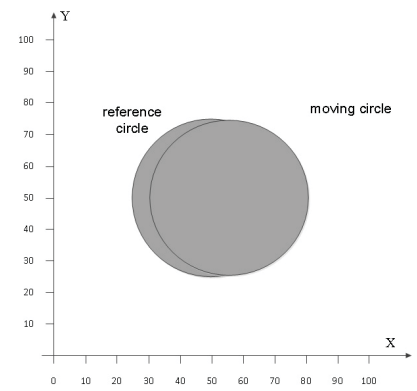

(a)

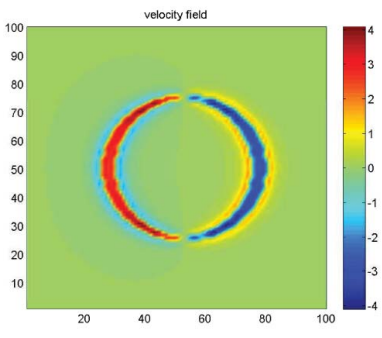

(b)

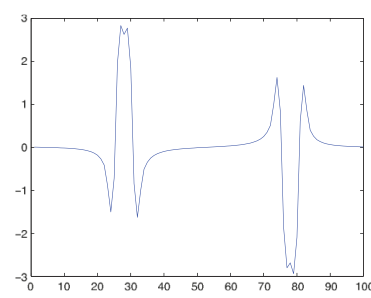

(c)

Fig. 5. (a) Two discs that are closer than those in Fig. 3. (b) Normal velocity field. (c) Normal velocity along the line passing the center of the disc ( $y=50$ ).

\section{E. Symmetric Diffeomorphic Registration With Dislocation Energy}

Diffeomorphism is a vigorously studied topic in the current image registration framework [12], [26], [27]. It promises to provide a differentiable transformation map with a differentiable inverse, making the transformation more physically reasonable and preserving properties such as smoothness of curves, surfaces or other features associated to anatomy. One of the most popular algorithms to solve diffeomorphic registration problem is Large Deformation Diffeomorphic Metric Matching (LDDMM) [11]. Diffeomorphism is represented as end point of paths parameterized by time-varying vector field defined on the tangent space of a convenient Riemannian manifold. It estimates the optimal transformation by constraining the transformation in the space of smooth velocity vector field $\mathrm{V}$ on domain $\Omega$ with the following form:

$$
\arg \min _{\mathbf{v}: \dot{\mathbf{h}}=\mathbf{v}_{t}(\mathbf{h})}\left(\int_{0}^{1}\left\|\mathbf{v}_{t}\right\|_{V}^{2} d t+\left|I_{m}(\mathbf{x}+\mathbf{h})-I_{r}(\mathbf{x})\right|^{2}\right) .
$$

This variational problem can be solved by using the EulerLagrange method. One superiority of LDDMM is its solid foundation in the mathematical framework. The existence of a solution is guaranteed by the integrability condition established for diffeomorphic image registration [28].

Avants et al. [15] extended the LDDMM framework to form a symmetric image normalization method ( $\mathrm{SyN})$. Their algorithm favors the natural symmetry in the path from $I_{m}$ to $I_{r}$ and $I_{r}$ to $I_{m}$, which makes the results independent of the choice of reference image and moving image. In a recent evaluation of image registration algorithms [29], the
SyN method's performance ranks high among the compared algorithms.

The variational optimization problem in SyN is defined as:

$$
\begin{aligned}
E= & \int_{t=0}^{0.5}\left(\left\|\mathbf{v}_{m}(\mathbf{x}, t)\right\|_{L}^{2}+\left\|\mathbf{v}_{r}(\mathbf{x}, t)\right\|_{L}^{2}\right) d t \\
& +\int_{\Omega}\left|I_{m}\left(\mathbf{x}+\mathbf{h}_{\mathbf{m}}\right)-I_{r}\left(\mathbf{x}+\mathbf{h}_{\mathbf{r}}\right)\right|^{2} d \Omega,
\end{aligned}
$$

subject to each $\mathbf{h}_{i} \in \operatorname{Diff}_{0}(i=m, r)$ the solution of: $d \mathbf{h}_{i}(\mathbf{x}, t) / d t=\mathbf{v}_{i}\left(\mathbf{h}_{i}(\mathbf{x}, t), t\right)$ with $\mathbf{h}_{i}(\mathbf{x}, 0)=\mathbf{I d}$ and $\mathbf{h}_{i}^{-1}\left(\mathbf{h}_{i}\right)=\mathbf{I d}, \mathbf{h}_{i}\left(\mathbf{h}_{i}^{-1}\right)=\mathbf{I d}$. The functional norm, $\|\cdot\|_{L}$, induces regularity on the velocity field via a linear differential operator, as explained in [15].

By taking variation of this function with respect to $\mathbf{h}_{m}$ at time 0.5 and $\mathbf{h}_{r}$ at time 0.5, two Euler-Lagrange equations can be derived to solve the optimization problem as:

$$
\begin{aligned}
\mathbf{F}_{m}= & \nabla \mathbf{h}_{m}(\mathbf{x}, 0.5) E \\
= & 2 L \mathbf{v}_{m}(\mathbf{x}, 0.5)-2\left(I_{r}\left(\mathbf{x}+\mathbf{h}_{\mathbf{r}}\right)\right. \\
& \left.-I_{m}\left(\mathbf{x}+\mathbf{h}_{\mathbf{m}}\right)\right)\left|D \mathbf{h}_{\mathbf{m}}\right| \nabla I_{m}\left(\mathbf{x}+\mathbf{h}_{\mathbf{m}}\right), \\
\mathbf{F}_{r}= & \nabla \mathbf{h}_{r}(\mathbf{x}, 0.5) E \\
= & 2 L \mathbf{v}_{r}(\mathbf{x}, 0.5)+2\left(I_{r}\left(\mathbf{x}+\mathbf{h}_{\mathbf{r}}\right)\right. \\
& \left.-I_{m}\left(\mathbf{x}+\mathbf{h}_{\mathbf{m}}\right)\right)\left|D \mathbf{h}_{\mathbf{r}}\right| \nabla I_{r}\left(\mathbf{x}+\mathbf{h}_{\mathbf{r}}\right) .
\end{aligned}
$$

As stated in [15], this SyN framework is flexible enough to adapt to different similarity metrics. With this SyN framework, our proposed similarity energy function is extended to make the transformation diffeomorphic. The proposed energy 
function is extended to Equation 22

$$
\begin{gathered}
E=\int_{t=0}^{0.5}\left(\left\|\mathbf{v}_{m}(\mathbf{x}, t)\right\|_{L}^{2}+\left\|\mathbf{v}_{r}(\mathbf{x}, t)\right\|_{L}^{2}\right) d t+\frac{1}{8 \pi} \int_{\Omega} d \mathbf{x} \\
\int_{\Omega} \frac{\nabla\left(I_{r}\left(\mathbf{x}+\mathbf{h}_{\mathbf{r}}\right)-I_{m}\left(\mathbf{x}+\mathbf{h}_{\mathbf{m}}\right)\right) \cdot \nabla\left(I_{r}\left(\mathbf{x}^{\prime}+\mathbf{h}_{\mathbf{r}}^{\prime}\right)-I_{m}\left(\mathbf{x}^{\prime}+\mathbf{h}_{\mathbf{m}}^{\prime}\right)\right)}{r} d \mathbf{x}^{\prime} .
\end{gathered}
$$

The driving forces applied to the images to minimize the total energy, which follow the derivation in Appendix A, are shown as follows,

$$
\begin{aligned}
\mathbf{F}_{m}= & \nabla \mathbf{h}_{m}(\mathbf{x}, 0.5) E \\
= & 2 L \mathbf{v}_{m}(\mathbf{x}, 0.5)-\frac{1}{4 \pi} \int_{\Omega} \frac{\mathbf{r} \cdot \nabla\left(I_{r}\left(\mathbf{x}+\mathbf{h}_{\mathbf{r}}\right)-I_{m}\left(\mathbf{x}+\mathbf{h}_{\mathbf{m}}\right)\right)}{r^{3}} d \mathbf{x} \\
& \times\left|D \mathbf{h}_{\mathbf{m}}\right| \frac{\nabla I_{m}}{\left|\nabla I_{m}\right|}, \\
\mathbf{F}_{r}= & \nabla \mathbf{h}_{r}(\mathbf{x}, 0.5) E \\
= & 2 L \mathbf{v}_{r}(\mathbf{x}, 0.5)+\frac{1}{4 \pi} \int_{\Omega} \frac{\mathbf{r} \cdot \nabla\left(I_{r}\left(\mathbf{x}+\mathbf{h}_{\mathbf{r}}\right)-I_{m}\left(\mathbf{x}+\mathbf{h}_{\mathbf{m}}\right)\right)}{r^{3}} d \mathbf{x} \\
& \times\left|D \mathbf{h}_{\mathbf{r}}\right| \frac{\nabla I_{r}}{\left|\nabla I_{r}\right|} .
\end{aligned}
$$

Regarding the implementation, smoothing using the Gaussian kernel can be applied on the images to smear out some noise. Hereinafter, without explicit specification, the image gradient in this paper is taking the gradient after smoothing by the Gaussian filter. The optimal solution of the energy function with respect to the velocity can be iteratively calculated by a standard gradient descent scheme. In the $k$ th iteration, the velocity is updated by:

$$
\mathbf{v}_{i}^{k+1}=\mathbf{v}_{i}^{k}-t \mathbf{F}_{i}
$$

It can be perceived from these formulas that the force driving the deformation in our proposed method (Equation 23(24)) is not only related to the similarity (in the numerator), but also the distance (in the denominator). It is actually driving the object boundary of the moving image to the closest and most similar object boundary in the reference image. Such a force can be viewed as a comprehensive force taking the global information of the entire image domain into consideration, which greatly enlarges the capture range.

As the force is computed through the integral over the entire image domain, computation of the force can bring a major computational issue. However, this computational issue can be solved by using the fast Fourier transform (FFT). The integral part of the force can be considered as the convolution of the two vector functions $\nabla\left(I_{r}-I_{m}\right)$ and $\nabla \frac{1}{r}$ with a factor $\frac{1}{4 \pi}$. Using FFT, the convolution of two vector functions can be transformed into the multiplication of the two Fourier transformed vector functions. This can greatly reduce the computational complexity in the implementation. The numerical implementation details of this transformation can be found in [25].

\section{F. Comparison Between Different Similarity Metrics}

Within the registration framework, several similarity metrics can be chosen as discussed in the introduction. The SSD metric accumulates the intensity difference of each voxel between two involved images and produces the driving force for each voxel. The CC metric is a slightly less restrictive metric which allows a linear relationship between the corresponding intensities. The $\mathrm{CC}$ depends on the estimates of the local image average and variance in a local region. It can enlarge the captured region as compared with SSD. However, it is still confined in a local neighborhood region. Comparatively, the MI metric is a global metric based on the joint intensity histogram of two involved images. MI metric is not a voxel-wise similarity metric, thus in most cases can only be computed globally. In order to make it applicable for non-rigid registration, some methods find the deformation on a local scale [30], [31] with the help of control points and parameterized deformation model. However, with the locality in the MI estimate increases, its statistical reliability decreases. As investigated in [32], the MI metric can perform well in affine registration, but it is still not optimal for non-rigid registration as compared with SSD and CC metrics. The proposed metric in this paper produces voxel-wise force like SSD and CC, but the force of each voxel computed by Equation 23(24) is an integration of the gradient information within the entire image domain. The new metric can be considered as a metric that can produce the voxelwise force with a global view. This property helps achieve the largest possible capture region, and thus reduce the time spent finding correspondences. We also provide a theoretical proof for explaining the fast convergence property of the proposed metric in Appendix B.

\section{EXPERIMENTAL RESULTS}

\section{A. Database and Implementation Issues}

In this section, we evaluate the proposed method on 3D magnetic resonance (MR) brain images. All MR images were obtained from the Internet Brain Segmentation Repository (IBSR V2.0) which includes the T1-weighted MR brain images of 18 normal subjects, available at ${ }^{1}$. The 18 brain scans have been positionally normalized into the Talairach orientation and have been processed by the CMA bias field correction routines. The resolution of each scan is $256 \times$ $256 \times 128$ voxels. The voxel size is $0.9375 \times 0.9375 \times$ $1.5 \mathrm{~mm}^{3}$ for 8 scans, $1.0 \times 1.0 \times 1.5 \mathrm{~mm}^{3}$ for 6 scans and $0.8371 \times 0.8371 \times 1.5 \mathrm{~mm}^{3}$ for 4 scans. Besides the T1-weight scans, the brain structure segmentation results are also provided from IBSR. These segmentation results serve as the ground truths for our experiment validation. In the following sections, the registration accuracy, convergence property and computation time are experimentally compared between the proposed method and the SyN method with SSD metric and CC metric. For the SyN method, the source code is available on the website [33]. The implementation of our method is based on the registration framework of SyN. The FFTW library obtained from [34] is used for fast FFT computation in our method. All the experiments were implemented on a PC with Intel(R) Core(TM) 2 Duo CPU and 4 GB RAM.

\footnotetext{
${ }^{1}$ Available at http://www.cma.mgh.harvard.edu/ibsr/.
} 
(a)

(b)

(c)

(d)

(e)

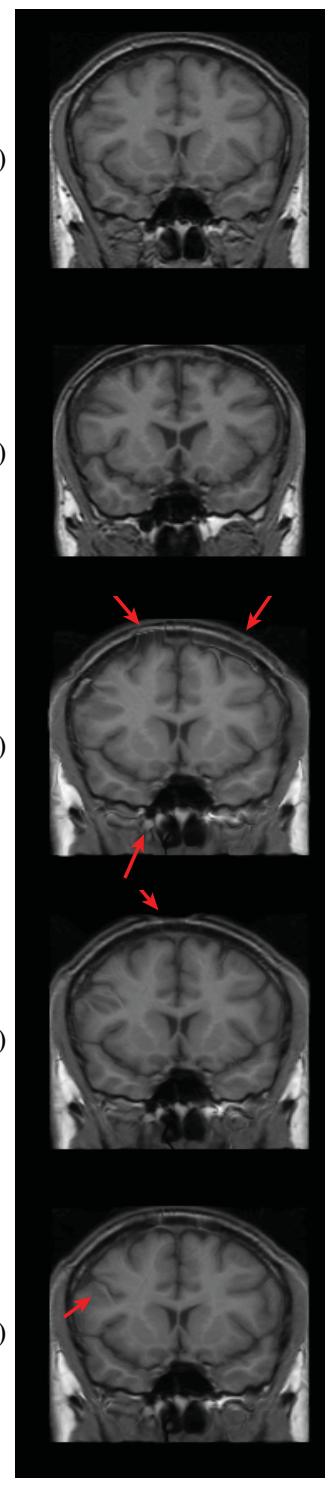

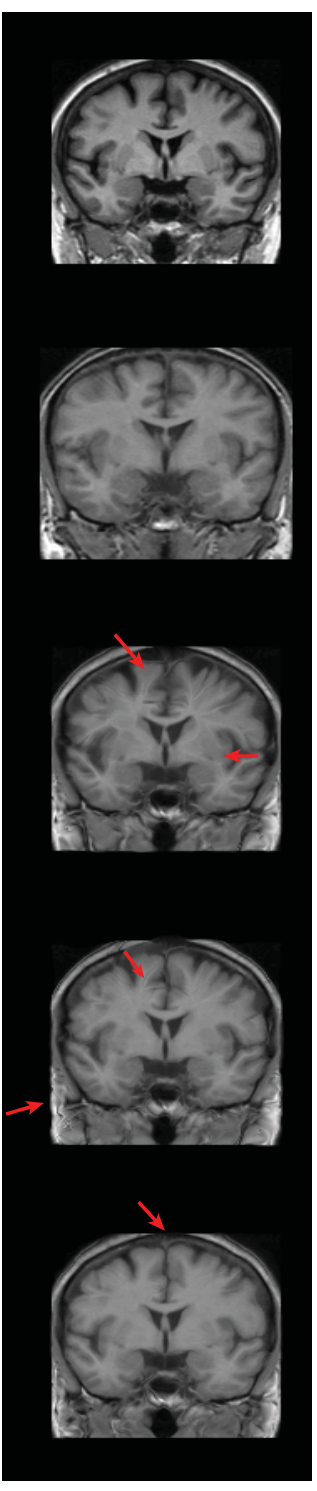
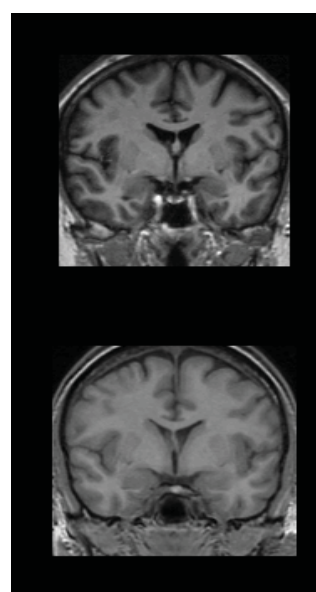

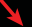

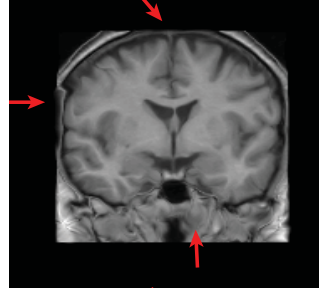

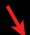

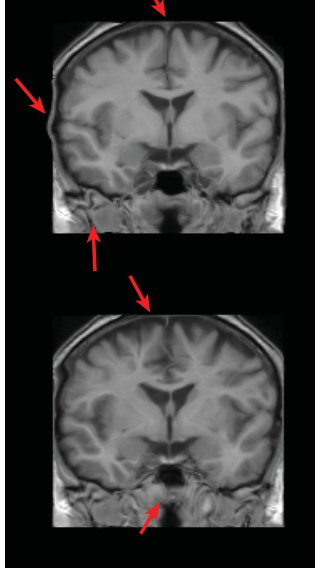

Fig. 6. 2-D slices obtained from the 3-D MR brain image experiments. Each column shows one pair of registration experiment using different methods. (a) Reference image. (b) Moving image. Registered images obtained by (c) SyN SSD method, (d) SyN CC method, and the (e) proposed method. The obvious mismatched regions are highlighted by arrows.

\section{B. Registration Accuracy}

To quantitatively and qualitatively evaluate the registration accuracy and show the convergence property and computation time of the proposed method, 3D image registration experiments were conducted on the 18 subject MR brain scans. Among the 18 brain volumes, one was served as the reference and the other 17 subjects were moving images. We performed 9 sets of experiments on the 18 subjects. We randomly chose 9 data sets in the IBSR database to serve as the reference images one-by-one and performed the pairwise registrations on the remaining 17 subjects. In the 3D experiments, affine registration was applied first. Then each method was applied with an four-level multi-resolution scheme for non-rigid registration. We allowed 100 iterations at the first three levels and 20 iterations at the full resolution. All methods were implemented using the same optimization framework. The gradient time steps were set to 0.5 and the regularization is
Gauss[3,0], which indicates that the velocity field is smoothed by a Gaussian filter with variance of $3 \times$ the image spacing. Figure 6 shows three examples of registration results for visual inspection. For illustration, 2D slices extracted from the registered 3D volumes obtained by the three compared methods together with the corresponding reference images and moving images before registration are shown. Each column represents one pair of example. It can be observed that the registered subject images using the proposed method (the bottom row in Figure 6) are most similar to the reference images, although there are still some misaligned structures, especially sulci and gyri. It is mainly due to their high similarities between the nearby structures.

Apart from a visual inspection, a quantitative evaluation was also conducted based on the volume overlap between the structure volumes of reference images and the transformed structure volumes of moving images and the distance error between the structure surfaces of the reference image and transformed 
TABLE I

Each Row Lists the Mean and Standard Deviation Values of the Volume Overlap Measurements of WM, GM, CSF, Ven, Pu, Th, And Cu of the Remaining 17 3-D Nonrigid Image Registration EXPeriments Based on One Reference Image. A, B, C, ANd D Representing the Results Before Registration, Using Syn SSD Method, Syn CC Method and Our Proposed Method, Respectively

\begin{tabular}{|c|c|c|c|c|c|c|c|c|c|c|c|c|c|c|c|c|c|c|c|c|c|c|c|c|c|c|c|c|c|}
\hline \multirow[t]{2}{*}{ Ref } & \multicolumn{4}{|c|}{ WM } & \multicolumn{4}{|c|}{ GM } & \multicolumn{4}{|c|}{ CSF } & \multicolumn{4}{|c|}{ Ven } & \multicolumn{4}{|c|}{ Th } & \multicolumn{4}{|c|}{$\mathrm{Pu}$} & \multicolumn{5}{|c|}{$\mathrm{Cu}$} \\
\hline & & B & $\mathrm{C}$ & D & & B & $\mathrm{C}$ & D & & B & $\mathrm{C}$ & D & & B & C & D & A & B & $\mathrm{C}$ & D & A & B & $\mathrm{C}$ & D & A & B & $\mathrm{C}$ & E & D \\
\hline & 0,1 & 0.66 & 0.70 & 0.74 & 0.49 & 0.67 & 0.72 & 0.74 & 0.34 & 0.67 & 0.71 & 0.74 & 0.21 & 0.39 & 0.49 & 0.51 & 0.64 & 0.65 & 0.70 & 0.75 & 0.47 & 0.44 & 0.67 & 0.68 & 0.41 & 0.56 & 60. & .64 & 0.67 \\
\hline & 04 & 0.08 & 0.04 & 0.03 & 0.06 & 0.10 & 0.05 & 0.05 & 0.09 & 0.07 & 0.03 & 0.03 & 0.08 & 0.08 & 0.03 & 0.04 & 0.05 & 0.10 & 0.07 & 0.03 & 0.10 & 0.20 & 0.04 & 0.04 & 0.10 & 0.10 & & .05 & 0.04 \\
\hline \multirow{2}{*}{2} & 0.44 & 0.69 & 0.69 & 0.73 & 0.45 & 0.69 & 0.70 & 0.73 & 0.28 & 0.64 & 0.65 & 0.68 & 0.23 & 0.58 & 0.60 & 0.63 & 0.89 & 0.67 & 0.65 & 0.71 & 0.53 & 0.57 & 70.69 & 0.70 & 0.46 & 0.57 & 70. & .62 & 0.67 \\
\hline & 04 & 0.09 & 0.07 & 0.07 & 0.06 & 0.10 & 0.08 & 0.07 & 0.07 & 0.10 & 0.10 & 0.09 & 0.13 & 0.10 & 0.09 & 0.08 & 0.07 & 0.10 & 0.12 & 0.06 & 0.12 & 0.17 & 70.08 & 0.07 & 0.09 & 0.16 & & .09 & 0.09 \\
\hline \multirow{2}{*}{3} & 0.45 & 0.65 & 0.67 & 0.72 & 0.47 & 0.61 & 0.63 & 0.69 & 0.31 & 0.57 & 0.65 & 0.67 & 0.22 & 0.50 & 0.60 & 0.63 & 0.64 & 0.56 & 0.67 & 0.72 & 0.54 & 0.31 & 10.64 & 0.68 & 0.43 & 0.4 & & .60 & 0.65 \\
\hline & 0.05 & 0.09 & 0.08 & 0.07 & 0.08 & 0.12 & 0.10 & 0.07 & 0.09 & 0.11 & 0.08 & 0.08 & 0.16 & 0.13 & 0.10 & 0.10 & 0.05 & 0.12 & 0.08 & 0.04 & 0.12 & 0.18 & 0.10 & 0.09 & 0.08 & 0.16 & & .09 & 0.08 \\
\hline \multirow{2}{*}{4} & 0.40 & 0.61 & 0.71 & 0.71 & 0.39 & 0.66 & 0.69 & 0.69 & 0.22 & 0.65 & 0.67 & 0.68 & 0.25 & 0.56 & 0.58 & 0.58 & 0.53 & 0.69 & 0.70 & 0.76 & 0.38 & 0.63 & 30.64 & 0.69 & 0.32 & 0.5 & & .60 & 0.66 \\
\hline & 0.06 & 0.12 & 0.09 & 0.09 & 0.08 & 0.09 & 0.11 & 0.08 & 0.08 & 0.10 & 0.09 & 0.09 & 0.12 & 0.13 & 0.16 & 0.16 & 0.08 & 0.09 & 0.07 & 0.04 & 0.14 & 0.14 & 40.15 & 0.11 & 0.10 & 0.13 & & .12 & 0.08 \\
\hline & 0.43 & 0.62 & 0.72 & 0.72 & 0.45 & 0.64 & 0.70 & 0.70 & 0.32 & 0.63 & 0.75 & 0.76 & 0.24 & 0.47 & 0.62 & 0.64 & 0.64 & 0.64 & 0.73 & 0.76 & 0.51 & 0.58 & 0.72 & 0.72 & 0.46 & 0.5 & & .65 & 0.68 \\
\hline & 0.05 & 0.12 & 0.07 & 0.08 & 0.07 & 0.09 & 0.09 & 0.07 & 0.09 & 0.10 & 0.08 & 0.08 & 0.08 & 0.20 & 0.07 & 0.14 & 0.07 & 0.10 & 0.05 & 0.04 & 0.14 & 0.16 & 50.10 & 0.09 & 0.10 & 0.16 & & .09 & 0.10 \\
\hline \multirow{2}{*}{ ( } & 0.44 & 0.68 & 0.69 & 0.72 & 0.47 & 0.73 & 0.72 & 0.75 & 0.31 & 0.67 & 0.67 & 0.71 & 0.28 & 0.60 & 0.61 & 0.64 & 0.65 & 0.73 & 0.73 & 0.75 & 0.52 & 0.67 & 70.75 & 0.75 & 0.43 & 0.6 & & .61 & 0.65 \\
\hline & 0.04 & 0.08 & 0.08 & 0.06 & 0.06 & 0.09 & 0.10 & 0.07 & 0.06 & 0.10 & 0.09 & 0.09 & 0.10 & 0.11 & 0.10 & 0.09 & 0.07 & 0.10 & 0.10 & 0.06 & 0.12 & 0.14 & 40.09 & 0.08 & 0.09 & 0.13 & & 12 & 0.09 \\
\hline \multirow{2}{*}{7} & 0.39 & 0.64 & 0.68 & 0.70 & 0.42 & 0.67 & 0.70 & 0.73 & 0.22 & 0.51 & 0.54 & 0.56 & 0.26 & 0.55 & 0.62 & 0.63 & 0.66 & 0.71 & 0.71 & 0.76 & 0.49 & 0.60 & 0.69 & 0.72 & 0.41 & 0.5 & & .48 & 0.52 \\
\hline & 0.04 & 0.09 & 0.08 & 0.07 & 0.08 & 0.12 & 0.10 & 0.07 & 0.05 & 0.09 & 0.11 & 0.09 & 0.09 & 0.14 & 0.09 & 0.09 & 0.07 & 0.11 & 0.11 & 0.09 & 0.10 & 0.16 & 50.08 & 0.07 & 0.08 & 0.16 & & .16 & 0.16 \\
\hline \multirow[t]{2}{*}{8} & 0.35 & 0.61 & 0.66 & 0.67 & 0.34 & 0.68 & 0.67 & 0.70 & 0.22 & 0.65 & 0.66 & 0.67 & 0.21 & 0.54 & 0.57 & 0.59 & 0.55 & 0.70 & 0.71 & 0.77 & 0.30 & 0.60 & 0.61 & 0.70 & 0.35 & 0.5 & & .56 & 0.63 \\
\hline & 0.06 & 0.07 & 0.07 & 0.07 & 0.10 & 0.06 & 0.06 & 0.06 & 0.07 & 0.10 & 0.07 & 0.06 & 0.07 & 0.16 & 0.11 & 0.10 & 0.09 & 0.12 & 0.06 & 0.03 & 0.13 & 0.13 & 30.13 & 0.09 & 0.07 & 70.1 & & .11 & 0.08 \\
\hline \multirow[t]{2}{*}{9} & 0.41 & 0.55 & 0.65 & 0.69 & 0.39 & 0.59 & 0.60 & 0.63 & 0.31 & 0.68 & 0.73 & 0.79 & 0.27 & 0.52 & 0.60 & 0.60 & 0.58 & 0.66 & 0.63 & 0.75 & 0.43 & 0.58 & 0.63 & 0.71 & 0.30 & 0.4 & & .54 & 0.66 \\
\hline & 0.06 & 0.13 & 0.13 & 0.08 & 0.08 & 0.11 & 0.09 & 0.08 & 0.10 & 0.13 & 0.07 & 0.05 & 0.14 & 0.12 & 0.12 & 0.11 & 0.09 & 0.10 & 0.15 & 0.04 & 0.14 & 0.12 & 20.12 & 0.10 & 0.11 & 0.1 & & .18 & 0.11 \\
\hline \multirow{2}{*}{ Average } & 0.42 & 0.63 & 0.68 & 0.71 & 0.43 & 0.66 & 0.68 & 0.71 & 0.28 & 0.63 & 0.67 & 0.70 & 0.24 & 0.52 & 0.59 & 0.61 & 0.64 & 0.67 & 0.70 & 0.75 & 0.46 & 0.55 & 50.67 & 0.71 & 0.39 & 0.5 & 50. & .59 & 0.64 \\
\hline & 0.03 & 0.04 & 0.02 & 0.02 & 0.04 & 0.04 & 0.04 & 0.03 & 0.05 & 0.05 & 0.06 & 0.06 & 0.02 & 0.06 & 0.04 & 0.04 & 0.10 & 0.04 & 0.03 & 0.02 & 0.08 & 0.10 & 0.04 & 0.02 & 0.05 & 0.0 & & .05 & 0.04 \\
\hline
\end{tabular}

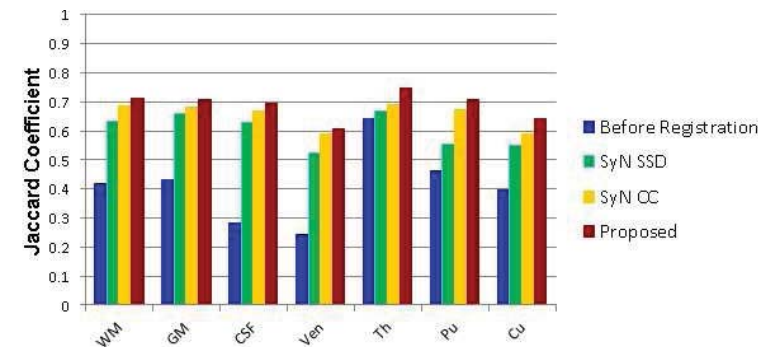

Fig. 7. Accuracy comparison of the registration results in terms of average Jaccard Coefficient. The average results obtained before registration, from SyN SSD method, SyN CC method, and the proposed method are represented by blue, green, yellow, red bars, respectively.

moving images. Seven brain structures, i.e., gray matter (GM), white matter (WM), cerebrospinal fluid (CSF), ventricle (Ven), putamen $(\mathrm{Pu})$, thalamus $(\mathrm{Th})$ and caudate $(\mathrm{Cu})$, are picked as they are most important brain structures in clinical diagnosis and commonly studied structures in brain anatomy literatures [35]-[38]. The Jaccard Coefficient metric [39] was used to measure the similarity between two registrations of the same region. For two registered regions $\mathrm{A}$ and $\mathrm{B}$, the Jaccard Coefficient is defined as: $P=\frac{|A \cap B|}{|A \cup B|}$, where $|$.$| defines the area$ of region under consideration. The segmentation ground truth was built based on the segmentation labels provided by IBSR. Table I lists the average structure-wise Jaccard Coefficient results over the 17 subjects in the each of the 9 sets of experiments. For better examination of the results, the average results of the 9 sets of experiments are plotted in Figure 7. As volume overlap measure does not explicitly account for

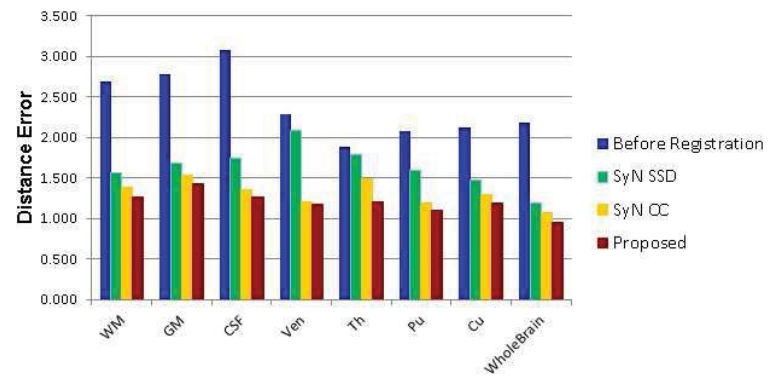

Fig. 8. Accuracy comparison of the registration results in terms of average distance error (DE). The average results obtained before registration, from SyN SSD method, SyN CC method, and the proposed method are represented by blue, green, yellow, red bars, respectively.

boundary discrepancies, we also measure the average distance error DE [29]: $D E=\frac{1}{P} \sum_{p=1}^{P} \operatorname{mindist}\left(S_{r p}, S_{m}\right)$. DE measures the average minimum distance from each reference surface point $S_{r p}$ to the entire set of points extracted from the moving surface $S_{m}$. We measure the DE values for each of the 7 brain structures as well as for the entire set of structure surface as a whole. Table II lists the DE results for each of the 9 sets of experiments and Figure 8 plots the average results of the 9 sets of experiments. From the results in Table I, II and Figure 7, 8, we find that the proposed method outperforms the other two methods for all brain structures consistently.

\section{Convergence Property and Computation Time}

Apart from registration accuracy, we have also compared the convergence property of the proposed method with other 
TABLE II

Each Row Lists the Mean and Standard Deviation Values of the Distance Error of WM, GM, CSF, Ven, Pu, Th, Cu, and the Whole

Brain of the Remaining 17 3-D NonRigid IMAge Registration EXPeriments Based on One Reference IMAGE. A, B, C, AND D

Representing the Results Before Registration, Using Syn SSD Method, SyN CC Method and Our Proposed Method, Respectively

\begin{tabular}{|c|c|c|c|c|c|c|c|c|c|c|c|c|c|c|c|c|c|c|c|c|c|c|c|c|c|c|c|c|c|c|c|c|}
\hline \multirow[t]{2}{*}{ Ref } & \multicolumn{4}{|c|}{ WM } & \multicolumn{4}{|c|}{ GM } & \multicolumn{4}{|c|}{ CSF } & \multicolumn{4}{|c|}{ Ven } & \multicolumn{4}{|c|}{ Th } & \multicolumn{4}{|c|}{$\mathrm{Pu}$} & \multicolumn{4}{|c|}{$\mathrm{Cu}$} & \multicolumn{4}{|c|}{ WholeBrain } \\
\hline & A & B & C & D & A & B & C & D & A & B & C & D & A & B & C & D & A & B & C & D & A & B & C & D & A & B & C & D & A & B & C & D \\
\hline \multirow{2}{*}{1} & 2.25 & 1.37 & 1.33 & 1.16 & 2.33 & 1.47 & 1.45 & 1.32 & 2.41 & 1.13 & 1.16 & 0.96 & 2.25 & 1.08 & 0.91 & 0.91 & 1.93 & 1.67 & 1.92 & 1.41 & 1.65 & 1.35 & 1.07 & 1.03 & 1.59 & 1.06 & 1.11 & 0.97 & 1.76 & 1.01 & 0.98 & 0.84 \\
\hline & 2.01 & 1.35 & 1.42 & 1.33 & 2.12 & 1.44 & 1.54 & 1.47 & 2.11 & 1.46 & 1.45 & 1.23 & 1.69 & 1.15 & 1.16 & 1.15 & 1.58 & 1.57 & 1.63 & 1.27 & 1.33 & 1.27 & 1.12 & 0.95 & 1.40 & 1.03 & 1.11 & 0.95 & 1.99 & 1.31 & 1.38 & 1.29 \\
\hline \multirow[t]{2}{*}{2} & 2.67 & 1.77 & 1.65 & 1.32 & 2.77 & 1.89 & 1.82 & 1.49 & 2.43 & 1.23 & 1.08 & 0.86 & 2.41 & 1.51 & 1.17 & 1.08 & 1.65 & 1.69 & 1.66 & 1.24 & 1.62 & 2.03 & 1.02 & 1.16 & 1.91 & 1.34 & 1.18 & 1.04 & 2.18 & 1.36 & 1.30 & 0.98 \\
\hline & 2.51 & 1.71 & 1.84 & 1.47 & 2.61 & 1.80 & 1.98 & 1.60 & 2.25 & 1.63 & 1.50 & 1.14 & 2.07 & 1.65 & 1.82 & 1.67 & 1.41 & 1.68 & 1.59 & 1.20 & 1.28 & 1.55 & 1.00 & 0.97 & 1.63 & 1.20 & 1.14 & 1.01 & 2.50 & 1.67 & 1.82 & 1.43 \\
\hline \multirow[t]{2}{*}{3} & 3.52 & 1.79 & 1.26 & 1.34 & 3.70 & 1.95 & 1.43 & 1.52 & 3.81 & 1.22 & 1.05 & 0.95 & 2.40 & 4.01 & 2.54 & 2.58 & 2.93 & 1.37 & 1.21 & 1.17 & 3.02 & 1.13 & 1.41 & 1.20 & 3.02 & 0.99 & 1.03 & 0.96 & 3.04 & 1.41 & 0.98 & 1.01 \\
\hline & 3.58 & 2.67 & 1.91 & 2.03 & 3.76 & 2.77 & 2.07 & 2.16 & 3.23 & 2.07 & 2.04 & 1.83 & 2.20 & 4.05 & 2.68 & 2.75 & 2.33 & 1.34 & 1.18 & 1.17 & 2.24 & 1.10 & 1.53 & 1.00 & 2.45 & 1.08 & 1.22 & 1.01 & 3.60 & 2.58 & 1.87 & 1.96 \\
\hline \multirow[t]{2}{*}{4} & 2.36 & 1.54 & 1.02 & 1.02 & 2.43 & 1.70 & 1.21 & 1.14 & 2.98 & 3.20 & 1.17 & 1.18 & 2.12 & 4.85 & 0.62 & 0.58 & 1.74 & 2.96 & 1.02 & 0.94 & 2.03 & 2.14 & 1.03 & 1.15 & 1.97 & 2.36 & 1.12 & 1.10 & 1.87 & 1.19 & 0.73 & 0.73 \\
\hline & 2.29 & 2.16 & 1.36 & 1.59 & 2.38 & 2.35 & 1.49 & 1.78 & 3.10 & 4.62 & 2.69 & 2.75 & 1.70 & 2.28 & 0.81 & 1.35 & 1.50 & 2.72 & 1.02 & 0.98 & 1.56 & 2.43 & 1.08 & 1.17 & 1.71 & 2.66 & 1.40 & 1.37 & 2.26 & 2.13 & 1.30 & 1.55 \\
\hline \multirow{2}{*}{5} & 2.23 & 1.25 & 1.31 & 1.07 & 2.26 & 1.36 & 1.44 & 1.23 & 2.71 & 1.39 & 1.31 & 1.16 & 1.71 & 1.52 & 1.00 & 0.85 & 1.45 & 1.28 & 1.25 & 1.05 & 1.60 & 1.41 & 0.91 & 0.89 & 1.69 & 1.06 & 1.16 & 1.07 & 1.72 & 0.90 & 0.99 & 0.76 \\
\hline & 2.04 & 1.35 & 1.55 & 1.22 & 2.10 & 1.44 & 1.66 & 1.35 & 2.80 & 2.30 & 1.94 & 1.88 & 1.53 & 1.52 & 1.43 & 1.11 & 1.24 & 1.13 & 1.15 & 1.01 & 1.24 & 1.12 & 0.98 & 0.87 & 1.39 & 0.99 & 1.01 & 1.00 & 1.99 & 1.28 & 1.50 & 1.17 \\
\hline \multirow[t]{2}{*}{6} & 2.33 & 1.68 & 1.37 & 1.21 & 2.37 & 1.77 & 1.49 & 1.35 & 3.88 & 2.35 & 2.33 & 2.30 & 2.10 & 2.09 & 1.00 & 0.99 & 1.44 & 1.86 & 1.74 & 1.39 & 1.77 & 1.83 & 1.45 & 1.35 & 1.80 & 1.18 & 1.92 & 2.03 & 1.81 & 1.28 & 1.05 & 0.89 \\
\hline & 2.06 & 1.49 & 1.55 & 1.35 & 2.12 & 1.57 & 1.65 & 1.44 & 4.16 & 4.01 & 3.55 & 3.69 & 1.77 & 1.65 & 1.45 & 1.30 & 1.24 & 1.39 & 1.66 & 1.34 & 1.43 & 1.41 & 1.33 & 1.42 & 1.49 & 1.17 & 1.58 & 1.49 & 2.02 & 1.47 & 1.52 & 1.31 \\
\hline \multirow{2}{*}{7} & 2.54 & 1.59 & 1.41 & 1.54 & 2.69 & 1.75 & 1.57 & 1.72 & 2.63 & 1.63 & 1.26 & 1.10 & 2.29 & 1.47 & 1.27 & 1.19 & 1.91 & 2.01 & 1.67 & 1.25 & 2.49 & 1.59 & 1.62 & 1.21 & 2.05 & 1.88 & 1.43 & 1.21 & 2.00 & 1.20 & 1.04 & 1.17 \\
\hline & 2.18 & 1.60 & 1.50 & 1.84 & 2.36 & 1.76 & 1.64 & 1.93 & 2.30 & 2.06 & 1.57 & 1.50 & 1.91 & 1.76 & 1.50 & 1.48 & 1.63 & 1.98 & 1.63 & 1.19 & 1.68 & 1.29 & 1.36 & 1.07 & 1.75 & 1.98 & 1.32 & 1.14 & 2.14 & 1.54 & 1.45 & 1.77 \\
\hline \multirow{2}{*}{8} & 3.15 & 1.74 & 1.81 & 1.47 & 3.30 & 1.90 & 2.02 & 1.67 & 2.83 & 1.30 & 0.93 & 0.81 & 2.12 & 1.30 & 1.34 & 1.49 & 2.23 & 1.55 & 1.35 & 0.99 & 2.33 & 1.46 & 1.04 & 0.85 & 2.64 & 1.66 & 1.21 & 0.99 & 2.65 & 1.34 & 1.52 & 1.17 \\
\hline & 3.02 & 2.24 & 2.20 & 3.36 & 3.18 & 2.41 & 2.37 & 3.46 & 2.36 & 1.55 & 1.35 & 1.44 & 2.03 & 1.37 & 1.73 & 1.73 & 1.80 & 1.50 & 1.23 & 0.98 & 1.65 & 1.30 & 0.98 & 0.86 & 2.07 & 1.57 & 1.33 & 1.21 & 3.03 & 2.21 & 3.26 & 2.15 \\
\hline \multirow{2}{*}{9} & 3.08 & 1.32 & 1.29 & 1.26 & 3.13 & 1.38 & 1.38 & 1.32 & 4.01 & 2.25 & 1.93 & 2.00 & 3.18 & 0.95 & 0.89 & 0.80 & 1.63 & 1.71 & 1.50 & 1.37 & 2.10 & 1.34 & 1.13 & 0.98 & 2.37 & 1.66 & 1.41 & 1.36 & 2.55 & 0.96 & 0.95 & 0.90 \\
\hline & 2.90 & 1.39 & 1.62 & 1.77 & 3.03 & 1.44 & 1.72 & 1.80 & 3.39 & 3.22 & 2.95 & 2.92 & 2.92 & 1.45 & 1.21 & 1.13 & 1.36 & 1.69 & 1.40 & 1.34 & 1.61 & 1.31 & 1.07 & 0.92 & 1.79 & 1.64 & 1.36 & 1.29 & 2.91 & 1.32 & 1.57 & 1.69 \\
\hline \multirow{2}{*}{ Average } & 2.68 & 1.56 & 1.38 & 1.27 & 2.77 & 1.68 & 1.53 & 1.42 & 3.08 & 1.74 & 1.36 & 1.26 & 2.29 & 2.09 & 1.19 & 1.16 & 1.88 & 1.79 & 1.48 & 1.20 & 2.07 & 1.58 & 1.19 & 1.09 & 2.12 & 1.46 & 1.29 & 1.19 & 2.17 & 1.18 & 1.06 & 0.94 \\
\hline & 2.51 & 1.77 & 1.66 & 1.77 & 2.63 & 1.89 & 1.79 & 1.89 & 2.86 & 2.55 & 2.11 & 2.04 & 1.98 & 1.88 & 1.53 & 1.52 & 1.57 & 1.67 & 1.39 & 1.16 & 1.56 & 1.42 & 1.16 & 1.03 & 1.748 & 1.48 & 1.27 & 1.16 & 2.24 & 1.72 & 1.74 & 1.59 \\
\hline
\end{tabular}

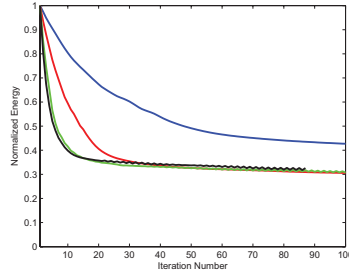

(a)

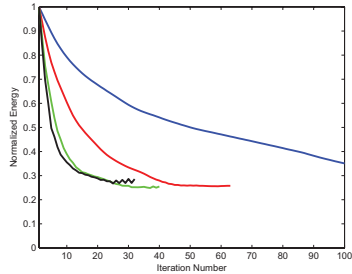

(e)

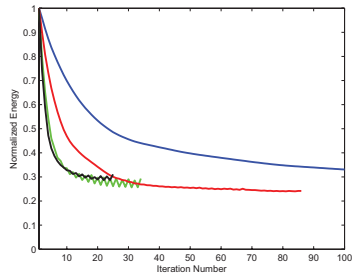

(i)

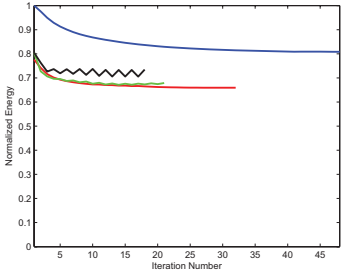

(b)

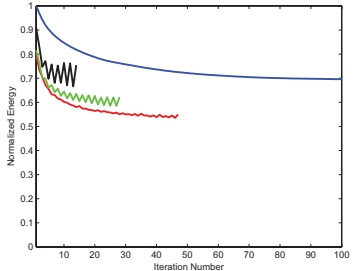

(f)

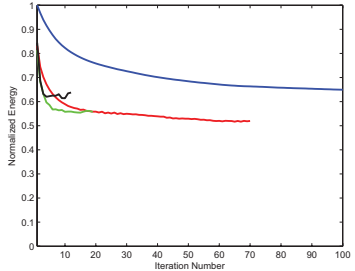

(j)

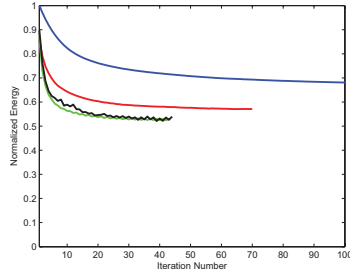

(c)

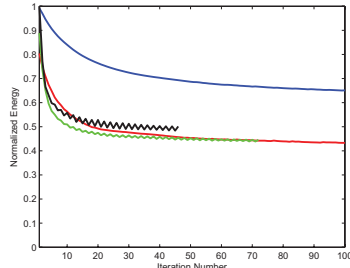

(g)

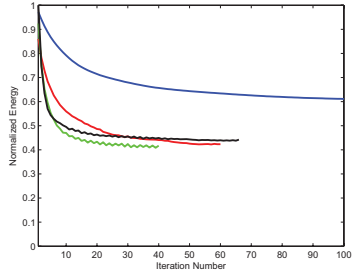

(k)

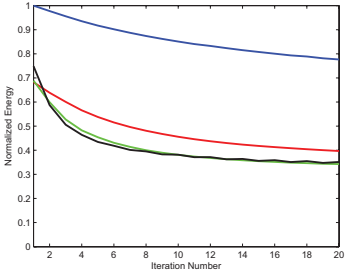

(d)

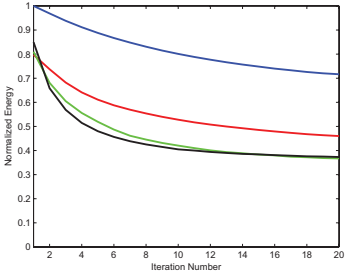

(h)

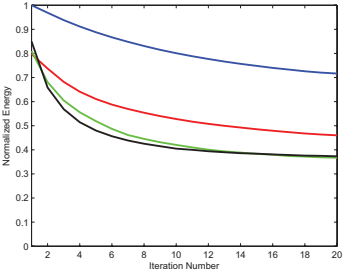

(1)

Fig. 9. Normalized energies with respect to the iteration number using three compared methods. Each curve represents one choice of gradient parameter. Blue curve: 0.1. Red curve: 0.25. Green curve: 0.5. Black curve: 0.7. Each row shows the results for one metric in all the four levels. (a) SSD level 1. (b) SSD level 2. (c) SSD level 3. (d) SSD level 4. (e) CC level 1. (f) CC level 2. (g) CC level 3. (h) CC level 4. (i) Proposed level 1. (j) Proposed level 2. (k) Proposed level 3. (l) Proposed level 4.

methods. For a registration algorithm, the convergence property is mainly determined by two factors: one is the optimization strategy and the other is the energy function. We used the gradient descent method for optimization, which was implemented in [33]. The method uses a fixed gradient parameter (i.e., the time step size). We have compared four choices of gradient parameter (i.e., 0.1, 0.25, 0.5, 0.7) and conducted experiments to observe the effect of the gradient time step. Figure 9 shows the variation of energy with respect to the iteration number for each of the three compared methods. As the multi-resolution scheme was applied, each sub-figure shows the convergence property comparison for 


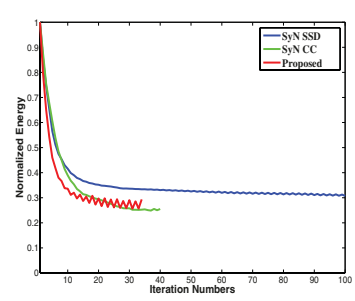

(a)

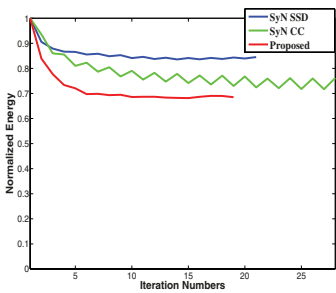

(b)

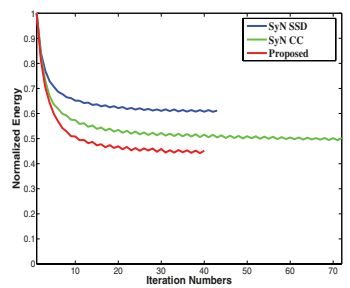

(c)

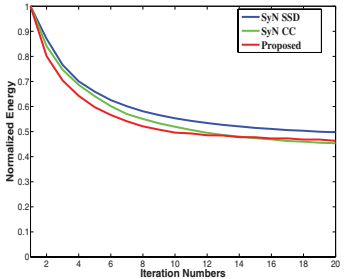

(d)

Fig. 10. Normalized energies with respect to the iteration number in one image registration experiment using three compared methods. Each curve represents one metric: blue curve for SSD, green curve for CC, and red curve for the proposed metric. (a) Level 1. (b) Level 2. (c) Level 3. (d) Level 4.

each of the four levels. The curves of the energy variation have been normalized with respect to the maximal energy values in each level. Here the convergence rate is defined as the speed at which a convergent sequence approaches its limit. In practice, for an iterative method, typically higher rate of convergence means fewer iterations are needed to yield a useful approximation or larger slope of energy variation curve if not converged yet when reaching the maximal iteration number (e.g., in our fourth level). It can be observed that generally the increase of the gradient descent parameter results in higher convergence rate. However, as it is also pointed out in [32], very large step size will result in energy oscillation and possibly trapped in local minima. But very small step size results in slow convergence. To make compromise between convergence rate and accuracy, we find 0.5 is the optimal choice for the three metrics. As in the fourth levels, the converge energy profiles are the lowest with this choice for the three metrics.

Then our main focus in the second factor, that is, the energy function. We fix the optimization strategy and compare the convergence property impacted by the similarity metrics. Figure 10 shows the variation of energy with respect to the iteration number in one image registration example. In order to make comparison between different energy function values taken by different methods, the curves of the energy variation have been normalized with respect to their initial energy values. It is observed from Figure 10, in the lower-level stages (the first three levels), the optimizations for three metrics converge within the given maximum iteration number. Our proposed metric provides the highest rate of convergence, as it requires the least number of iterations to converge. In the final level, three energy functions do not converge within the maximum iteration number. We can compare the convergence rate based on the slope of energy variation curve. It can be observed that in the early stage of this level (the first 10 iterations), our metric has the fastest convergence rate in terms of the slope of energy variation. But in the later stage, the speed becomes slower due to the inherent property of our metric. Our metric tries to find the correspondences in the whole image domain. It thus uses a more aggressive way to find correspondences and locates the region of interest (ROI) for finding the correspondences quickly because of its longrange interaction. Comparatively, SSD and $\mathrm{CC}$ method find the correspondences in the local neighborhood region, resulting a more conservative way. When energy approaches the global minima (convergence point), the differences between different metrics become smaller, as the search regions for all metrics are now confined in the local region. Therefore the superiority of our metric is more obvious in the early stage of optimization.

With regard to the running time, we compared the average running times relative to that of the proposed method as the actual running times can be different on different machines and for different subjects. The average times for one pair of non-rigid registration experiment are 1 (the proposed method, on average 2 hours depending on the subjects), 1.5 (SyN SSD method) and 4 (SyN CC method). The less computational cost of our method indicates the fast convergence property possessed by the new energy function as illustrated in Figure 10, which also experimentally validates the conclusion made in Appendix B. In general, the proposed method needs smaller number of iterations to converge as compared with SyN methods. Moreover, the utilization of FFT implementation in our method further shortens the time cost for each iteration. Therefore, the total running time is relatively shorter. Comparatively, SyN CC method needs the longest computational time because it needs to calculate the crosscorrelation coefficient in local neighborhood region of each voxel which greatly increases the computational time in each iteration.

\section{CONCLUSION}

In this paper, we have proposed a new non-rigid image registration method which is inspired by the crystal dislocation theory. Based on the orientation-dependent and long-range interaction between dislocations in crystals, we develop a new energy function for the image registration problem. The underlying driving force is different to other registration methods because it is a comprehensive force which is derived based on the global information of the entire image domain. This property endows the optimization of this new framework with a faster rate of convergence. Moreover, the energy term (i.e., the similarity metric) has been added into a diffeomorphism framework to make the transformation diffeomorphic. Through comparison with other methods, the convergence property is theoretically proved. Based on the theoretical proof, it is concluded that the rate of convergence of the proposed method is faster. This conclusion is also validated through experiments. In the experiments, apart from the convergence property, the registration accuracy is also tested with $3 \mathrm{D}$ MR brain images of the IBSR database. It is qualitatively and quantitatively demonstrated that with the new similarity metric, which is 
derived from the dislocation theory, the registration results are more accurate.

\section{APPENDIX A}

In this appendix, we use the dislocation physics model to derive the formulation of the new energy function and the force driving image registration.

When transferring from the dislocation model to the image registration problem, the $\delta(\gamma)$ function in Equation 6 is equivalent to,

$$
\delta(\gamma)=|\nabla I| \delta(z),
$$

where $\delta(z)$ is the one dimensional delta function of $z$. With the directions of object boundaries defined in Equations 13 and 14 , we now have

$$
\begin{aligned}
\delta(\gamma) \tau_{\mathbf{r}} & =\nabla I_{r} \times \mathbf{k} \delta(z), \\
\delta(\gamma) \tau_{\mathbf{m}} & =-\nabla I_{m} \times \mathbf{k} \delta(z) .
\end{aligned}
$$

Using a linear combination of Equations $\mathrm{A} 2$ and $\mathrm{A} 3$ for the right-hand side of Equation 6, the constraint equation now becomes

$$
\nabla \times \mathbf{w}=\nabla\left(I_{r}-I_{m}\right) \times \mathbf{k} \delta(z) .
$$

In dislocation model, we allow $\gamma(s)$ to move to minimize the energy stated in Equation 12, the dynamics equation in the steepest descent direction is

$$
\mathbf{F}=\mathbf{w} \times \boldsymbol{\tau} .
$$

Moreover, through Equation 9, it can be verified that $w_{1}(x, y, 0)=w_{2}(x, y, 0)=0$. As such, using Equation A5 and $w_{1}(x, y, 0)=w_{2}(x, y, 0)=0$, the force driving the moving curve is

$$
\mathbf{F}=w_{3} \mathbf{k} \times\left(-\frac{\nabla I_{m}}{\left|\nabla I_{m}\right|} \times \mathbf{k}\right)=-w_{3} \frac{\nabla I_{m}}{\left|\nabla I_{m}\right|}=-\mathbf{w} \cdot \mathbf{k} \frac{\nabla I_{m}}{\left|\nabla I_{m}\right|} .
$$

Then using Equations 9 and A4, from Equation A6, we have

$$
\begin{aligned}
\mathbf{F} & =\frac{1}{4 \pi} \int_{\Omega} \frac{\mathbf{r} \times\left(\nabla\left(I_{r}-I_{m}\right) \times \mathbf{k}\right) \cdot \mathbf{k}}{r^{3}} d \mathbf{x} \frac{\nabla I_{m}}{\left|\nabla I_{m}\right|} \\
& =-\frac{1}{4 \pi} \int_{\Omega} \frac{\mathbf{r} \cdot \nabla\left(I_{r}-I_{m}\right)}{r^{3}} d \mathbf{x} \frac{\nabla I_{m}}{\left|\nabla I_{m}\right|} .
\end{aligned}
$$

Under this force, the boundary curve in the moving image will move to find the corresponding boundary in the reference image by minimizing the total energy, which is

$$
E=\frac{1}{8 \pi} \int_{\Omega} d \mathbf{x} \int_{\Omega} \frac{\nabla\left(I_{r}-I_{m}\right) \cdot \nabla\left(I_{r}-I_{m}\right)}{r} d \mathbf{x}^{\prime} .
$$

\section{APPENDIX B}

As it is shown in Section II-E, the variational problem of the image registration can be solved iteratively by applying the Equation 25. The existence of a solution for $\mathbf{v}$ calculated by Equation 25 can be guaranteed by the integrability condition established for the diffeomorphic image registration [28]. Here the property of this solution will be discussed, and the convergence rate of the optimization problem will be presented.
Lemma 1: Suppose that $I_{r}, I_{m} \in L^{2}\left(\mathbb{R}^{2}\right)$, then $\mathbf{F}$ in Equations 20, 21 and Equations 23, 24 are the Lipschitzcontinuous and the Lipschitz constants $C$ which depend on $I_{r}, I_{m}$ [28], [40].

Theorem 1: Suppose that $I_{r}, I_{m} \in L^{2}\left(\mathbb{R}^{2}\right)$, then for all $\mathbf{v}(0) \in H$, where $H$ is the separable Hilbert space $(H=$ $L^{2}\left(\mathbb{R}^{2}\right) \times L^{2}\left(\mathbb{R}^{2}\right)$ ), there exists a unique solution of $\mathbf{v}$.

Proof: Inspired by [40], which presents the existence and uniqueness of solutions for a parabolic system, the existence and uniqueness of the solution for $\mathbf{v}$ is proved as follows.

$\mathbf{v}$ is calculated through the updating Equation 25. According to the Picard-Lindelof Theorem, Equation 25 has a classical solution:

$$
\mathbf{v}(t)=\mathbf{v}(0)+\int_{0}^{t}-\mathbf{F}(\mathbf{v}(s)) d s .
$$

Assume $\mathbf{v}_{1}(t)$ and $\mathbf{v}_{2}(t)$ are solutions of Equation B1 for initial conditions $\mathbf{v}_{1}(0)$ and $\mathbf{v}_{2}(0)$, respectively.

According to the Lipschitz continuity of $\mathbf{F}$ shown in Lemma 1, with $\mathrm{C}$ as the Lipschitz constant of $\mathbf{F}$, we have,

$$
\left\|\mathbf{F}\left(\mathbf{v}_{1}(s)\right)-\mathbf{F}\left(\mathbf{v}_{2}(s)\right)\right\|_{H} \leq C\left\|\mathbf{v}_{1}(s)-\mathbf{v}_{2}(s)\right\|_{H} .
$$

Then from B1, we have

$$
\begin{aligned}
\left\|\mathbf{v}_{1}(t)-\mathbf{v}_{2}(t)\right\|_{H} \leq & \left\|\mathbf{v}_{1}(0)-\mathbf{v}_{2}(0)\right\|_{H} \\
& +C \int_{0}^{t}\left\|\mathbf{v}_{1}(s)-\mathbf{v}_{2}(s)\right\|_{H} d s .
\end{aligned}
$$

With the Gronwall-Bellman lemma, it has,

$$
\left\|\mathbf{v}_{1}(t)-\mathbf{v}_{2}(t)\right\|_{H} \leq e^{C t}\left\|\mathbf{v}_{1}(0)-\mathbf{v}_{2}(0)\right\|_{H},
$$

which yields the uniqueness of the solution if it exists.

Now consider the Banach space defined by

$$
B=\left\{\mathbf{v}: \sup _{t \geq 0}\|\mathbf{v}(t)\|_{H} e^{-K t}<\infty\right\},
$$

endowed with the norm $\|\mathbf{v}(t)\|_{B}=\sup _{t \geq 0}\|\mathbf{v}(t)\|_{H} e^{-K t}$, where $K$ is a constant.

Let $\phi$ be defined by

$$
\phi(\mathbf{v})(t)=\mathbf{v}(0)+\int_{0}^{t}-\mathbf{F}(\mathbf{v}(s)) d s .
$$

If $K>C$, then $\phi(B) \subset B$, and $\phi$ is $\frac{C}{K}$-Lipschitz since

$$
\begin{aligned}
\left\|\phi\left(\mathbf{v}_{1}\right)-\phi\left(\mathbf{v}_{2}\right)\right\|_{B} & =\sup _{t \geq 0}\left\|\phi\left(\mathbf{v}_{1}\right)(t)-\phi\left(\mathbf{v}_{2}\right)(t)\right\|_{H} e^{-K t} \\
& \leq \sup _{t \geq 0} \int_{0}^{t} C\left\|\mathbf{v}_{1}(s)-\mathbf{v}_{2}(s)\right\|_{H} d s e^{-K t} \\
& \leq \sup _{t \geq 0}\left\|\mathbf{v}_{1}-\mathbf{v}_{2}\right\|_{B} e^{-K t} \int_{0}^{t} e^{K s} d s \\
& \leq \sup _{t \geq 0} \frac{C}{K}\left\|\mathbf{v}_{1}-\mathbf{v}_{2}\right\|_{B} e^{-K t}\left(e^{K t}-1\right) \\
& \leq \frac{C}{K}\left\|\mathbf{v}_{1}-\mathbf{v}_{2}\right\|_{B} .
\end{aligned}
$$

It can be deduced that $\phi$ is a contraction. With the Banach's fixed point theorem, there exists a unique $\mathbf{v}$, s.t. $\phi(\mathbf{v})=\mathbf{v}$, which is the generalized solution for $\mathbf{v}$. 
Theorem 2: Based on the Theorem 1, the convergence rate of the optimization problem is related with the Lipschitz constant $C$ of $\mathbf{F}$.

Proof: According to the Banach's fixed point theorem, let $(X, d)$ be a non-empty complete metric space. Let $\phi: X \rightarrow X$ be a contraction mapping on $X$, i.e., there is a nonnegative real number $q<1$ such that, $d(\phi(x), \phi(y)) \leq q d(x, y)$, for all $x, y$ in $X$. Then the map $\phi$ admits one and only one fixed point $x^{*}$.

Through the derivation in Theorem 1,

$$
\left\|\phi\left(\mathbf{v}_{1}\right)-\phi\left(\mathbf{v}_{2}\right)\right\|_{B} \leq \frac{C}{K}\left\|\mathbf{v}_{1}-\mathbf{v}_{2}\right\|_{B},
$$

in which case, $q=\frac{C}{K}$.

Next we will prove that $q$ is actually related to the convergence rate. As the fixed point can be found as follows: start with an arbitrary $x_{0}$, the iterative sequence $x_{n}=\phi\left(x_{n-1}\right)$ converges and its limit is $x^{*}$. The speed of convergence can be calculated by:

$$
d\left(x^{*}, x_{n}\right) \leq \frac{q^{n}}{1-q} d\left(x_{1}, x_{0}\right),
$$

equivalently

$$
d\left(x^{*}, x_{n+1}\right) \leq q d\left(x_{n}, x^{*}\right) .
$$

Through the definition of convergence, we get from B9 that $q$ is the convergence rate. The smaller the $q$, the faster the convergence. Then in the registration framework, $q=\frac{C}{K}$ and $K$ is not concerned with the algorithm itself. Therefore, the convergence rate of the registration algorithm is proportional to $C$.

Theorem 3: The registration framework with the proposed similarity metric can converge faster than the SSD metric.

Proof: Define $\bar{I}_{r}=I_{r}\left(\mathbf{x}+\mathbf{h}_{\mathbf{r}}\right)$ and $\bar{I}_{m}\left(\mathbf{h}_{i}\right)=I_{m}\left(\mathbf{x}+\mathbf{h}_{i}\right)$ with $\mathbf{h}_{i}(i=1,2) \in H$.

For SSD metric, with the Equation 20,

$$
\mathbf{F}_{m}=2 L \mathbf{v}-2\left(\bar{I}_{r}-\bar{I}_{m}(\mathbf{h})\right)|D \mathbf{h}| \nabla \bar{I}_{m}(\mathbf{h}) .
$$

Then

$$
\begin{aligned}
& \mathbf{F}_{m}\left(\mathbf{h}_{1}\right)-\mathbf{F}_{m}\left(\mathbf{h}_{2}\right) \\
&= 2 L \mathbf{v}_{1}-2 L \mathbf{v}_{2}-2\left(\bar{I}_{r}-\bar{I}_{m}\left(\mathbf{h}_{1}\right)\right)\left|D \mathbf{h}_{1}\right| \nabla \bar{I}_{m}\left(\mathbf{h}_{1}\right) \\
&+2\left(\bar{I}_{r}-\bar{I}_{m}\left(\mathbf{h}_{2}\right)\right)\left|D \mathbf{h}_{2}\right| \nabla \bar{I}_{m}\left(\mathbf{h}_{2}\right) \\
&= 2 L \mathbf{v}_{1}-2 L \mathbf{v}_{2}-2\left[\left(\bar{I}_{r}-\bar{I}_{m}\left(\mathbf{h}_{1}\right)\right)\right. \\
&\left.-\left(\bar{I}_{r}-\bar{I}_{m}\left(\mathbf{h}_{2}\right)\right)\right]\left|D \mathbf{h}_{1}\right| \nabla \bar{I}_{m}\left(\mathbf{h}_{1}\right) \\
&+2\left(\bar{I}_{r}-\bar{I}_{m}\left(\mathbf{h}_{2}\right)\right)\left(\left|D \mathbf{h}_{2}\right| \nabla \bar{I}_{m}\left(\mathbf{h}_{2}\right)-\left|D \mathbf{h}_{1}\right| \nabla \bar{I}_{m}\left(\mathbf{h}_{1}\right)\right) .
\end{aligned}
$$

We introduce some notations for functions defined on $\Omega$ : $\|f\|_{C^{0}}=\sup _{x \in \Omega}|f(x)|, \quad|f|_{C^{1}}=\sup _{x \in \Omega}|\nabla f(x)|,\|f\|_{C^{1}}=$ $\|f\|_{C^{0}}+|f|_{C^{1}}^{x \in \Omega}$, and the following Lipschitz norms:

$$
\begin{aligned}
& \|f\|_{C^{0,1}}=\|f\|_{C^{0}}+\sup _{x, y \in \Omega} \frac{|f(x)-f(y)|}{|x-y|}, \\
& \|f\|_{C^{1,1}}=\|f\|_{C^{1}}+\sup _{x, y \in \Omega} \frac{|\nabla f(x)-\nabla f(y)|}{|x-y|} .
\end{aligned}
$$

Therefore, from Equation B11, we have,

$$
\begin{aligned}
\left\|\mathbf{F}_{m}\left(\mathbf{h}_{1}\right)-\mathbf{F}_{m}\left(\mathbf{h}_{2}\right)\right\|_{L^{2}} \leq & 2 L\left\|\mathbf{v}_{1}-\mathbf{v}_{2}\right\|_{L^{2}} \\
& +2\left\|\bar{I}_{m}\right\|_{C^{1}}\left\|\bar{I}_{m}\right\|_{C^{0,1}}\left\|\mathbf{h}_{1}-\mathbf{h}_{2}\right\|_{L^{2}} \\
& +2\left\|\bar{I}_{m}\right\|_{C^{0}}\left\|\bar{I}_{m}\right\|_{C^{1,1}}\left\|\mathbf{h}_{1}-\mathbf{h}_{2}\right\|_{L^{2}} \\
& +2\left\|\bar{I}_{m}\right\|_{C^{1,1}}\left\|\bar{I}_{r}\right\|_{L^{\infty}}\left\|\mathbf{h}_{1}-\mathbf{h}_{2}\right\|_{L^{2}} .
\end{aligned}
$$

According to the Lemma 2.2 in [28], with a constant M,

$$
\left\|\mathbf{v}_{1}-\mathbf{v}_{2}\right\|_{L^{2}} \leq M\left\|\mathbf{h}_{1}-\mathbf{h}_{2}\right\|_{L^{2}}
$$

Then we can get:

$$
\left\|\mathbf{F}_{m}\left(\mathbf{h}_{1}\right)-\mathbf{F}_{m}\left(\mathbf{h}_{2}\right)\right\|_{L^{2}} \leq C_{S S D_{m}}\left\|\mathbf{h}_{1}-\mathbf{h}_{2}\right\|_{L^{2}},
$$

with the Lipschitz constant $C_{S S D_{m}}$ for $\mathbf{F}_{m}$,

$$
\begin{aligned}
C_{S S D_{m}}= & 2 L M+2\left\|\bar{I}_{m}\right\|_{C^{1}}\left\|\bar{I}_{m}\right\|_{C^{0,1}} \\
& +2\left\|\bar{I}_{m}\right\|_{C^{0}}\left\|\bar{I}_{m}\right\|_{C^{1,1}}+2\left\|\bar{I}_{m}\right\|_{C^{1,1}}\left\|\bar{I}_{r}\right\|_{L^{\infty}} .
\end{aligned}
$$

Applying the above procedure to the Equation 21, it can deduce the Lipschitz constant for $\mathbf{F}_{r}$ as follows,

$$
\begin{aligned}
C_{S S D_{r}}= & 2 L M+2\left\|\bar{I}_{r}\right\|_{C^{1}}\left\|\bar{I}_{r}\right\|_{C^{0,1}} \\
& +2\left\|\bar{I}_{r}\right\|_{C^{0}}\left\|\bar{I}_{r}\right\|_{C^{1,1}}+2\left\|\bar{I}_{r}\right\|_{C^{1,1}}\left\|\bar{I}_{m}\right\|_{L^{\infty}} .
\end{aligned}
$$

For the dislocation energy based similarity metric, with the Equation 23,

$$
\begin{aligned}
\mathbf{F}_{m} & =2 L \mathbf{v}-\frac{1}{4 \pi} \int_{\Omega} \frac{\mathbf{r} \cdot\left(\nabla \bar{I}_{r}-\nabla \bar{I}_{m}(\mathbf{h})\right)}{r^{3}} d \mathbf{x}|D \mathbf{h}| \frac{\nabla \bar{I}_{m}(\mathbf{h})}{\left|\nabla \bar{I}_{m}(\mathbf{h})\right|} \\
& =2 L \mathbf{v}-\left[\frac{1}{4 \pi}\left(\nabla \bar{I}_{r}-\nabla \bar{I}_{m}(\mathbf{h})\right)|D \mathbf{h}| \frac{\nabla \bar{I}_{m}(\mathbf{h})}{\left|\nabla \bar{I}_{m}(\mathbf{h})\right|}\right] * \nabla \frac{1}{r} .
\end{aligned}
$$

Then

$$
\begin{aligned}
& \mathbf{F}_{m}\left(\mathbf{h}_{1}\right)-\mathbf{F}_{m}\left(\mathbf{h}_{2}\right) \\
& =2 L \mathbf{v}_{1}-2 L \mathbf{v}_{2} \\
& -\frac{1}{4 \pi}\left[\left(\nabla \bar{I}_{r}-\nabla \bar{I}_{m}\left(\mathbf{h}_{1}\right)\right)\left|D \mathbf{h}_{1}\right| \frac{\nabla \bar{I}_{m}\left(\mathbf{h}_{1}\right)}{\left|\nabla \bar{I}_{m}\left(\mathbf{h}_{1}\right)\right|}\right. \\
& \left.\quad-\left(\nabla \bar{I}_{r}-\nabla \bar{I}_{m}\left(\mathbf{h}_{2}\right)\right)\left|D \mathbf{h}_{2}\right| \frac{\nabla \bar{I}_{m}\left(\mathbf{h}_{2}\right)}{\left|\nabla \bar{I}_{m}\left(\mathbf{h}_{2}\right)\right|}\right] * \nabla \frac{1}{r} \cdot \quad(\mathrm{B} 18) \\
& \begin{aligned}
\left\|\mathbf{F}_{m}\left(\mathbf{h}_{1}\right)-\mathbf{F}_{m}\left(\mathbf{h}_{2}\right)\right\|_{L^{2}} \\
\leq 2 L\left\|\mathbf{v}_{1}-\mathbf{v}_{2}\right\|_{L^{2}} \\
+\frac{1}{4 \pi} \|\left[\left(\left|D \mathbf{h}_{1}\right| \frac{\nabla \bar{I}_{m}\left(\mathbf{h}_{1}\right)}{\left|\nabla \bar{I}_{m}\left(\mathbf{h}_{1}\right)\right|}-\left|D \mathbf{h}_{2}\right| \frac{\nabla \bar{I}_{m}\left(\mathbf{h}_{2}\right)}{\left|\nabla \bar{I}_{m}\left(\mathbf{h}_{2}\right)\right|}\right) \nabla \bar{I}_{r}\right. \\
\left.\quad-\left(\left|D \mathbf{h}_{1}\right|\left|\nabla \bar{I}_{m}\left(\mathbf{h}_{1}\right)\right|-\left|D \mathbf{h}_{2}\right|\left|\nabla \bar{I}_{m}\left(\mathbf{h}_{2}\right)\right|\right)\right] * \nabla \frac{1}{r} \|_{L^{2}}
\end{aligned} .
\end{aligned}
$$

Using the Young's inequality:

$$
\|f * g\|_{r} \leq\|f\|_{p}\|g\|_{q},
$$


where $\frac{1}{p}+\frac{1}{q}=\frac{1}{r}+1$, and with the Lipschitz norms:

$$
\begin{aligned}
\|f\|_{C^{2,2}} & =\|f\|_{C^{2}}+\sup _{x, y \in \Omega} \frac{\left|\frac{\nabla f(x)}{|\nabla f(x)|}-\frac{\nabla f(y)}{|\nabla f(y)|}\right|}{|x-y|}, \\
\|f\|_{C^{2}} & =\sup _{x \in \Omega}\left|\frac{\nabla f(x)}{|\nabla f(x)|}\right| .
\end{aligned}
$$

We can obtain that,

$$
\begin{aligned}
\left\|\mathbf{F}_{m}\left(\mathbf{h}_{1}\right)-\mathbf{F}_{m}\left(\mathbf{h}_{2}\right)\right\|_{L^{2}} & \\
\leq 2 L\left\|\mathbf{v}_{1}-\mathbf{v}_{2}\right\|_{L^{2}}+\frac{1}{4 \pi} & \left(\left\|\bar{I}_{r}\right\|_{C^{1}}\left\|\bar{I}_{m}\right\|_{C^{2,2}}\left\|\mathbf{h}_{1}-\mathbf{h}_{2}\right\|_{L^{2}}\right. \\
& \left.+\left\|\bar{I}_{m}\right\|_{C^{1,1}}\left\|\mathbf{h}_{1}-\mathbf{h}_{2}\right\|_{L^{2}}\right)\left|\nabla \frac{1}{r}\right|_{L^{1}} .
\end{aligned}
$$

It leads to:

$$
\left\|\mathbf{F}_{m}\left(\mathbf{h}_{1}\right)-\mathbf{F}_{m}\left(\mathbf{h}_{2}\right)\right\|_{L^{2}} \leq C_{D i s_{m}}\left\|\mathbf{h}_{1}-\mathbf{h}_{2}\right\|_{L^{2}},
$$

with

$$
C_{D i s_{m}}=2 L M+\frac{1}{4 \pi}\left(\left\|\bar{I}_{r}\right\|_{C^{1}}\left\|\bar{I}_{m}\right\|_{C^{2,2}}+\left\|\bar{I}_{m}\right\|_{C^{1,1}}\right)\left|\nabla \frac{1}{r}\right|_{L^{1}} .
$$

Similarly, the following can be obtained,

$$
C_{D i s_{r}}=2 L M+\frac{1}{4 \pi}\left(\left\|\bar{I}_{m}\right\|_{C^{1}}\left\|\bar{I}_{r}\right\|_{C^{2,2}}+\left\|\bar{I}_{r}\right\|_{C^{1,1}}\right)\left|\nabla \frac{1}{r}\right|_{L^{1}} .
$$

Comparing with $C_{S S D_{m}}$ and $C_{S S D_{r}}$, it can be deduced that $C_{D i s_{m}}<C_{S S D_{m}}$ and $C_{D i s_{r}}<C_{S S D_{r}}$. With the conclusion of Theorem 2, it can be concluded that the registration framework with the crystal dislocation energy can converge faster than that with SSD metric.

\section{REFERENCES}

[1] J. Modersitzki, Numerical Methods for Image Registration. London, U.K.: Oxford Univ. Press, 2004.

[2] M. Holden, "A review of geometric transformations for nonrigid body registration," IEEE Trans. Med. Imag., vol. 27, no. 1, pp. 111-128, Jan. 2008.

[3] A. Evans, C. Beil, and S. Marrett, "Anatomical-functional correlation using an adjustable MRI-based region of interest atlas with positron emission tomography," J. Cereb. Blood Flow Metab., vol. 8, no. 4, pp. 513-530, Aug. 1988.

[4] C. Maurer, J. Fitzpatrick, and M. Wong, "Registration of head volume images using implantable fiducial markers," IEEE Trans. Med. Imag., vol. 16, no. 4, pp. 447-462, Aug. 1997.

[5] C. Davatzikos and J. Prince, "Brain image registration based on curve mapping," in Proc. IEEE Workshop Biomed. Image Anal., Jun. 1994, pp. 245-254.

[6] P. Besl and N. Mckay, "A method for registration of 3-D shapes," IEEE Trans. Pattern Anal. Mach. Intell., vol. 14, no. 2, pp. 239-256, Feb. 1992.

[7] D. Shen and C. Davatzikos, "HAMMER: Hierarchical attribute matching mechanism for elastic registration," IEEE Trans. Med. Imag., vol. 21, no. 11 , pp. 1421-1439, Nov. 2002.

[8] J. Hajnal, N. Saeed, and E. Soar, "A registration and interpolation procedure for subvoxel matching of serially acquired MR images," J. Comput. Assist. Tomogr., vol. 19, no. 2, pp. 289-296, 1995.

[9] J. Hajnal, N. Saeed, and A. Oatridge, "Detection of subtle brain changes using subvoxel registration and subtraction of serial MR images," $J$. Comput. Assist. Tomogr., vol. 19, no. 5, pp. 677-691, 1995.
[10] K. Friston, "Spatial registration and normalization of images," Human Brain Mapping, vol. 3, no. 3, pp. 165-189, 1995.

[11] M. Beg, M. Miller, A. Trouve, and L. Younes, "Computing large deformation metric mappings via geodesic flows of diffeomorphisms," Int. J. Comput. Vis., vol. 61, no. 2, pp. 139-157, 2005.

[12] T. Vercauteren, X. Pennec, P. Aymeric, and N. Ayache, "Non-parametric diffeomorphic image registration with the demons algorithm," in Proc. MICCAI, vol. 4792. 2007, pp. 319-326.

[13] J. Thirion, "Image matching as a diffusion process: An analogy with Maxwell's demons," Med. Image Anal., vol. 2, no. 3, pp. 243-260, 1998.

[14] B. Ardekani, S. Guckemus, A. Bachman, M. Hoptman, M. Wojtaszek, and J. Nierenberg, "Quantitative comparison of algorithms for intersubject registration of 3D volumetric brain MRI scans," J. Neurosci. Methods, vol. 142, no. 1, pp. 67-76, 2005.

[15] B. Avants, C. Epstein, M. Grossman, and J. Gee, "Symmetric diffeomorphic image registration with cross-correlation: Evaluating automated labeling of elderly and neurodegenerative brain," Med. Image Anal., vol. 12, no. 1, pp. 26-41, 2008.

[16] W. Wells, P. Viola, H. Atsumi, S. Nakajima, and R. Kikinis, "Multimodal volume registration by maximization of mutual information," Med. Image Anal., vol. 1, no. 1, pp. 35-52, 1996.

[17] F. Maes, D. Vandermeulen, and P. Suetens, "Medical image registration using mutual information," Proc. IEEE, vol. 91, no. 10, pp. 1699-1722, Oct. 2003.

[18] C. Studholme, D. L. G. Hill, and D. J. Hawkes, "An overlap invariant entropy measure of 3D medical image alignment," Pattern Recognit., vol. 32, no. 1, pp. 71-86, Jan. 1999.

[19] J. Hirth and J. Lothe, Theory of Dislocations, 2nd ed. New York: Wiley, 1982.

[20] C. Broit, "Optimal registration of deformed images," Ph.D. dissertation, Dept. Comput. Inf. Sci., Univ. Pennsylvania, Philadephia, 1981.

[21] G. Christensen, R. Rabbitt, and M. Miller, "Deformable templates using large deformation kinematics," IEEE Trans. Image Process., vol. 5, no. 10, pp. 1435-1447, Oct. 1996.

[22] H. Lester and S. Arridge, "A survey of hierarchical nonlinear medical image registration," Pattern Recognit., vol. 32, no. 1, pp. 129-149, 1999.

[23] B. Horn and B. Schunck, "Determining optical flow," Artif. Intell., vol. 17, nos. 1-3, pp. 185-203, 1981.

[24] R. Lardner, Mathematical Theory of Dislocations and Fracture. Toronto, ON, Canada: Univ. Toronto Press, 1974.

[25] Y. Xiang, A. Chung, and J. Ye, "An active contour model for image segmentation based on elastic interaction," J. Comput. Phys., vol. 219, no. 1 , pp. 455-476, 2006.

[26] M. Miller, "Computational anatomy: Shape, growth, and atrophy comparison via diffeomorphisms," Neuroimage, vol. 23, no. 1, pp. 19-33, 2004.

[27] J. Ashburner, "A fast diffeomorphic image registration algorithm," Neuroimage, vol. 38, no. 1, pp. 95-113, 2007.

[28] P. Dupuis, U. Grenander, and M. Miller, "Variational problems on flows of diffeomorphisms for image matching," Quart. Appl. Math., vol. 56, no. 3, pp. 587-600, 1998.

[29] A. Klein, J. Andersson, B. Ardekani, J. Ashburner, B. Avants, M. Chiang, G. Christensen, D. Collins, P. Hellier, J. Song, M. Jenkinson, C. Lepage, D. Rueckert, P. Thompson, T. Vercauteren, R. Woods, J. Mann, and R. Parsey, "Evaluation of 14 nonlinear deformation algorithms applied to human brain MRI registration," Neuroimage, vol. 46, no. 3, pp. 786-802, 2009.

[30] D. Mattes, D. Haynor, H. Vesselle, T. Lewellen, and W. Eubank, "PETCT image registration in the chest using free-form deformations," IEEE Trans. Med Imag., vol. 22, no. 1, pp. 120-128, Jan. 2003.

[31] C. Studholme, R. T. Constable, and J. S. Duncan, "Accurate alignment of functional EPI data to anatomical MRI using a physics-based distortion model," IEEE Trans. Med. Imag., vol. 19, no. 11, pp. 1115-1127, Nov. 2000.

[32] B. Avants, N. Tustison, G. Song, P. Cook, A. Klein, and J. Gee, "A reproducible evaluation of ANTs similarity metric performance in brain image registration," Neuroimage, vol. 54, no. 3, pp. 2033-2044, 2011.

[33] The Syn Code Website [Online]. Available: http://picsl.upenn.edu/ANTS/

[34] FFTW Website [Online]. Available: http://www.fftw.org/

[35] T. F. Cootes and C. Taylor, "Statistical models of appearance for medical image analysis and computer vision," Proc. SPIE Med. Imag., vol. 4322, pp. 236-248, Jul. 2001.

[36] K. Pohl, J. Fisher, W. Grimson, R. Kikinis, and W. Wells, "A Bayesian model for joint segmentation and registration," Neuroimage, vol. 31, no. 1 , pp. 228-239, 2006. 
[37] W. Crum, O. Camara, and D. Hill, "Generalized overlap measures for evaluation and validation in medical image analysis," IEEE Trans. Med. Imag., vol. 25, no. 11, pp. 1451-1461, Nov. 2006.

[38] M. Sdika, "A fast nonrigid image registration with constraints on the Jacobian using large scale constrained optimization," IEEE Trans. Med. Imag., vol. 27, no. 2, pp. 271-281, Feb. 2008.

[39] P. Jaccard, "The distribution of flora in the alpine zone," New Phytol., vol. 11, no. 2, pp. 37-50, 1912.

[40] L. Alvarez, J. Weickert, and J. Sánchez, "Reliable estimation of dense optical flow fields with large displacements," Int. J. Comput. Vis., vol. 39, no. 1, pp. 41-56, 2000.

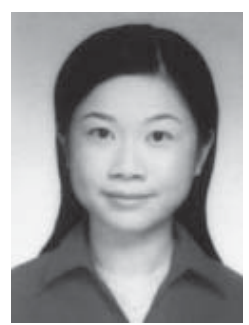

Yishan Luo received the B.Sc. and M.Sc. degrees in electronic engineering from Fudan University, Shanghai, China, in 2005 and 2008, respectively. She is currently pursuing the Ph.D. degree in computer science and engineering with the Hong Kong University of Science and Technology, Hong Kong.

Her current research interests include medical image registration and segmentation, and image enhancement.

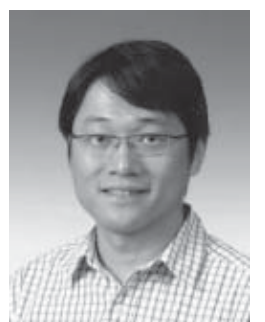

Albert C. S. Chung received the B.Eng. degree (Hons.) in computer engineering from the University of Hong Kong, Hong Kong, and the M.Phil. degree in computer science from the Hong Kong University of Science and Technology, Hong Kong, and the Doctoral Degree from the University of Oxford, Oxford, UK., in 1995, 1998, and 2001, respectively.

He joined the Medical Vision Laboratory, University of Oxford, as a Doctoral Research Student with a Croucher Foundation scholarship. $\mathrm{He}$ was a Visiting Scientist with the Artificial Intelligence Laboratory, Massachusetts Institute of Technology, Cambridge, in 2001. $\mathrm{He}$ is currently an Associate Professor with the Department of Computer Science and Engineering, Hong Kong University of Science and Technology. His current research interests include medical image analyses, image processing, and computer visions.

Dr. Chung was the recipient of the British Machine Vision Association Sullivan Thesis Award in 2002 for the Best Doctoral Thesis submitted to U.K. University in the field of computer or natural vision. 